\title{
Energy Gels: A Bio-Inspired Material Platform for Advanced Energy
}

\section{Applications}

Ye Shi ${ }^{a, \S}$, Jun Zhang ${ }^{a, \S}$, Lijia Pan ${ }^{\text {b }}$, Yi Shi ${ }^{\text {b,* }}$, Guihua Yu ${ }^{\text {a,* }}$

${ }^{a}$ Materials Science and Engineering Program and Department of Mechanical Engineering, The University of Texas at Austin, TX, 78712, USA.

${ }^{\mathrm{b}}$ Collaborative Innovation Center of Advanced Microstructures, School of Electronic Science and Engineering, Nanjing University, Nanjing 210093, China

${ }^{\S}$ These authors contribute equally.

*E-mails: ghyu@austin.utexas.edu; yshi@nju.edu.cn

\section{Summary}

Energy conversion and storage systems with high efficiency have attracted great research interest in recent years. To improve the performance of energy devices, nanomaterials with delicately controlled structures and interfaces have been applied. However, low-dimensional nanomaterials suffer from inhomogeneous aggregation, severe re-stacking, and the formation of bottlenecks and poor contacts during the processing and assembly, thus hindering the transport of electrons or ions in energy devices. Gel materials, a special class of bio-inspired materials, are emerging as promising candidates to overcome the drawbacks of low-dimensional nanomaterials. The 3D nanostructured gel network promotes electron transport along the backbone while facilitating the diffusion of ions through hierarchical pores, as well as providing high surface area for interfacial reactions. In addition, the properties of gels can be facilely tuned, thereby further extending their applications and improving their performance.

To date, various synthesis strategies have been developed for the preparation of gel materials, including carbon-based gels, conductive polymer gels, ionically conductive gels and inorganic gels. These gel materials have successfully served as 
electrode materials, electrolytes, self-supported current collectors, 3D binder systems, etc. in various kinds of energy conversion and storage applications, such as lithium batteries, supercapacitors, catalysts, and fuel cells. In this review, we summarize the synthesis of various electrically conductive gel materials, including carbon-based gels, conductive polymer gels, and ionically conductive gels and their applications in energy conversion and storage devices. We also provide the perspective on the future developments of these gel materials in energy-related fields.

\section{Introduction}

The development of high-performance energy conversion and storage systems is attracting intensive research interest because of the increasing demand for clean energy against the backdrop of energy crisis and environmental pollution throughout the world.[1-6] To improve the performance of energy devices, particular importance is attributed to both the invention of new materials and the delicate control of their structures, which has been demonstrated through successful applications of nanomaterials in recent decades.[7] However, simple nanostructured materials such as 0D particles, 1D nanowires and 2D nanosheets face problems such as inhomogeneous aggregation, severe re-stacking, and the formation of bottlenecks and poor contacts during the processing and assembly of materials and the operation of energy conversion and storage devices, hindering the transport of electrons or ions between domains and inside aggregation regions.[8]

To overcome the disadvantages of low-dimensional nanomaterials, the concept of assembling nanomaterials into macroscopic 3D architectures has been introduced in energy applications. $[9,10]$ The monoliths thus obtained not only inherit the properties of nanomaterials that result from their confined dimensions but also exhibit new features originating from the combination of a high internal reactive surface area and molecular transport pathways to the surface. Among the various macroscopic 3D architectures, gel-type materials are regarded as one promising candidate for energy-related applications because of their unique chemical, microstructural and mechanical features (Scheme 1).[8, 11] 


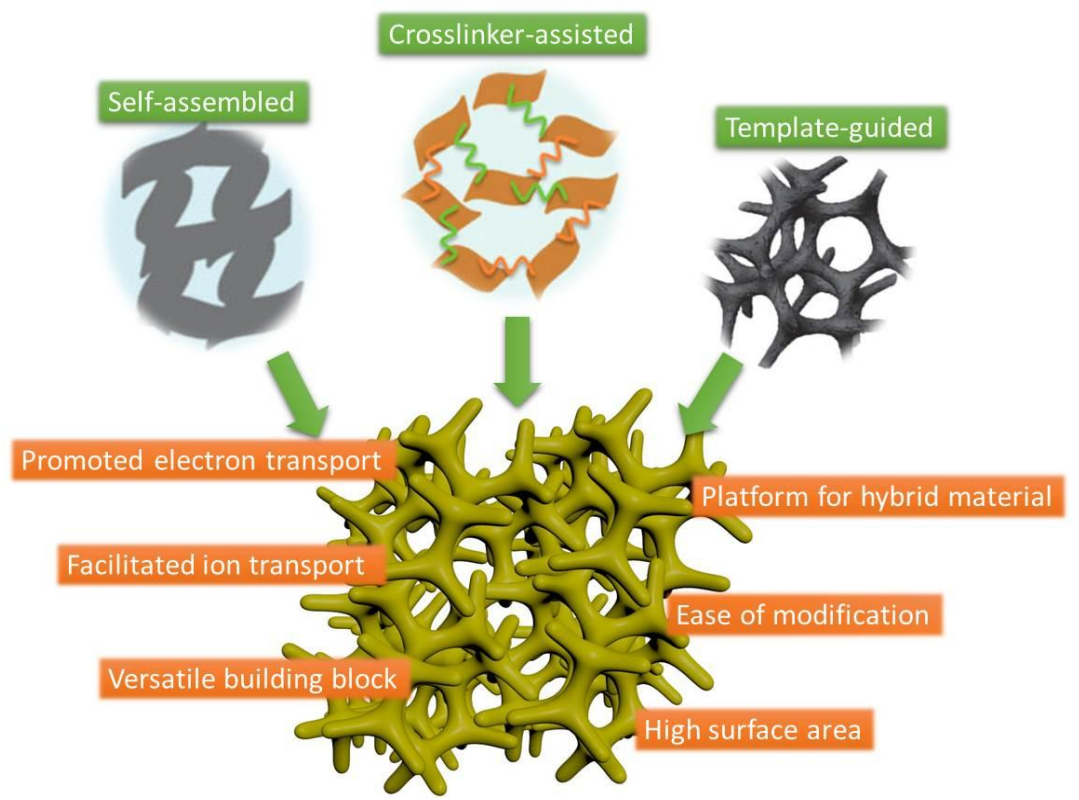

Scheme 1. Commonly used synthesis strategies for gel-based nanomaterials and the advantageous features of gels derived from their chemical and physical structures.

Gels are defined as cross-linked network systems that exhibit properties ranging from soft and weak to hard and tough. Conventionally, gels, especially hydrogels, mainly consist of homopolymer or copolymer chains crosslinked by either physical interactions or chemical bonds.[12] Physical gels are networks formed through non-covalent interactions, which usually lead to junction zones because separated polymer chains interact over a certain length but not in a point-to-point manner. Typical interactions include hydrophobic interactions, charge interactions and hydrogen bonds. When the crosslinking effects are covalent in nature, the gels thus formed can be regarded as chemical gels, which typically exhibit enhanced mechanical stability because covalent interactions are much stronger. Chemical crosslinking methods include radical polymerization, the chemical reaction of complementary groups, high-energy irradiation and the use of other functional crosslinking agents. With the development of gel chemistry and processing technologies, the building blocks for gel materials are no longer restricted to polymer chains. Various materials, including large organic molecules, carbon-based nanomaterials, metals, and other inorganic compounds, can be crosslinked to form 3D networks via strategies such as self-assembly, the use of crosslinkers, and 
template-guided methods (Scheme 1). The dimensions of building blocks can vary from $0 \mathrm{D}[13]$ to $1 \mathrm{D}[14,15]$ and even $2 \mathrm{D}[16]$; examples include quantum dots, nanowires and nanosheets, respectively. These newly developed gel materials are formed through physical or chemical interactions between the building blocks themselves or between the building blocks and crosslinking agents. Coulomb forces, $\pi-\pi$ interactions, hydrogen bonds, hydrophobic interactions and covalent bonds are the most common forces employed in the formation of these gel materials.
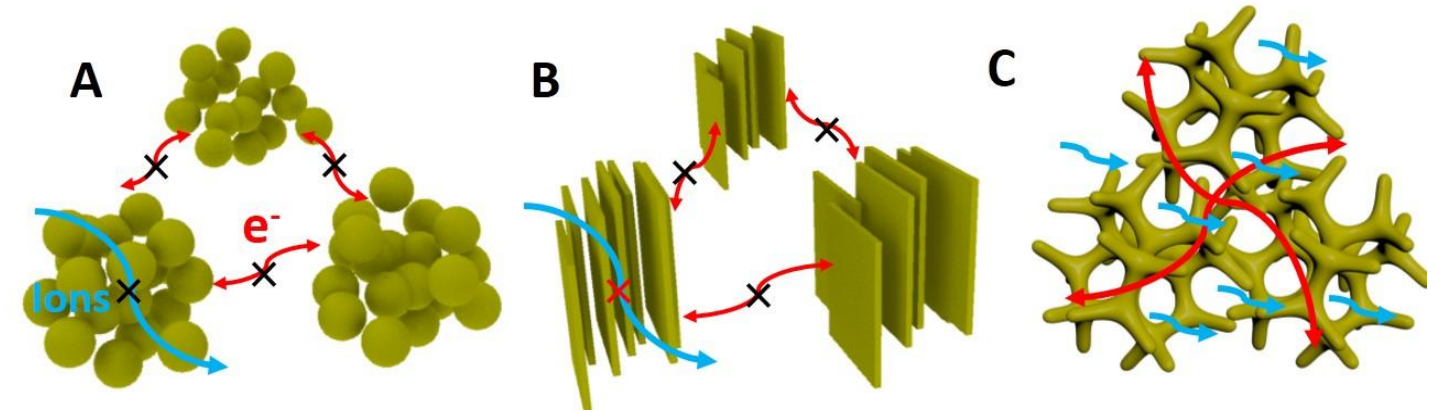

Scheme 2. (A and B) Low-dimensional nanomaterials may suffer from inhomogeneous aggregation and severe re-stacking during processing and device fabrication. (C) The 3D network of a gel can promote the transport of electrons along the backbone while facilitating the diffusion of ions through hierarchical pores.

Gels, especially hydrogels, have been widely studied and applied in bio-related fields, such as tissue engineering, controlled drug delivery, the development of medical and biological sensors, and diagnostic imaging.[17] The successful application of gel materials benefits from the advantageous features of their chemical and physical structures.[18] Gel networks have a high affinity for solvents but are prevented from dissolving because of their crosslinked nature. Solvents can penetrate between the chains, and the mechanical properties of gels can be modulated over a wide range. The gel matrix provides a large surface area and biocompatibility, allowing biologically active entities such as enzymes and cells to be incorporated into such networks and well dispersed by means of physical or chemical entrapment to produce hybrid materials. In addition, the molecular architecture and morphology of gels can be precisely controlled, resulting in tunable chemical and mechanical 
properties. Finally, gel networks can be made to interact with their environments in an intelligent manner via molecular tailoring. Various responsive gels have been synthesized that are capable of sensing and responding to external stimuli, such as $\mathrm{pH}$, light, mechanical stress, and temperature.

These appealing features of gel materials as demonstrated in bio-applications are also desirable in energy conversion and storage devices (Figure 1). Gel materials offer several important advantages: 1. The transport of both electrons and ions is crucial in energy devices. The 3D network of a gel can promote the transport of electrons along the backbone while facilitating the diffusion of ions through hierarchical pores (Scheme 2). The network structure can also mitigate the effects of strong interactions such as $\pi-\pi$ interactions, which can lead to the stacking of materials, and can accommodate the volume changes that occur during reactions, thereby suppressing the resulting damage to the transport pathways. 2. Energy conversion and storage involve physical interactions and/or chemical reactions at surfaces and interfaces; therefore, the surface area and surface chemistry of a material play very important roles in these processes.[7] Gel materials possess enhanced surface areas, which can provide large numbers of active sites for reactions, and their surfaces can be chemically modified. 3 . Hybrid materials also play important roles in high-performance energy devices. Gel materials offer an excellent platform for the development of various hybrid materials because the interconnected gel network acts as a framework to support other active materials and its porous structure permits the introduction of a second network. The hydrophilic nature of hydrogels also makes them compatible with bio-environments. 4. Gel materials exhibit beneficial mechanical properties, including tunable strength and high flexibility, which enable the development of flexible and stretchable devices. 5. Finally, the chemical, mechanical, and electrochemical properties of gels can be facilely tuned by adopting different functional crosslinkers and applying chemical modifications, thereby further extending their applications and improving their performance.

In the past decades, great progress has been achieved in the development of gel materials for energy applications, and several review papers have been published that 
have focused on specific materials, such as carbon-based gels,[8] conductive polymer gels,[11] and gel electrolytes.[19] A summary of the advantageous chemical and physical structural features shared by these gel materials and a discussion of the relationship between the properties of gels and the performance of energy devices are needed. In this review, we provide a full picture of the state-of-the-art gel materials that are available for energy applications and discuss various electrically conductive gel materials, including carbon-based gels, conductive polymer gels, as well as ionically conductive gels. The general synthesis strategies for these gel materials are summarized, and their applications in energy conversion and storage devices, such as lithium ion batteries, supercapacitors, catalysts, and fuel cells, are discussed. A perspective on the future developments of gel materials in energy-related fields is also provided.

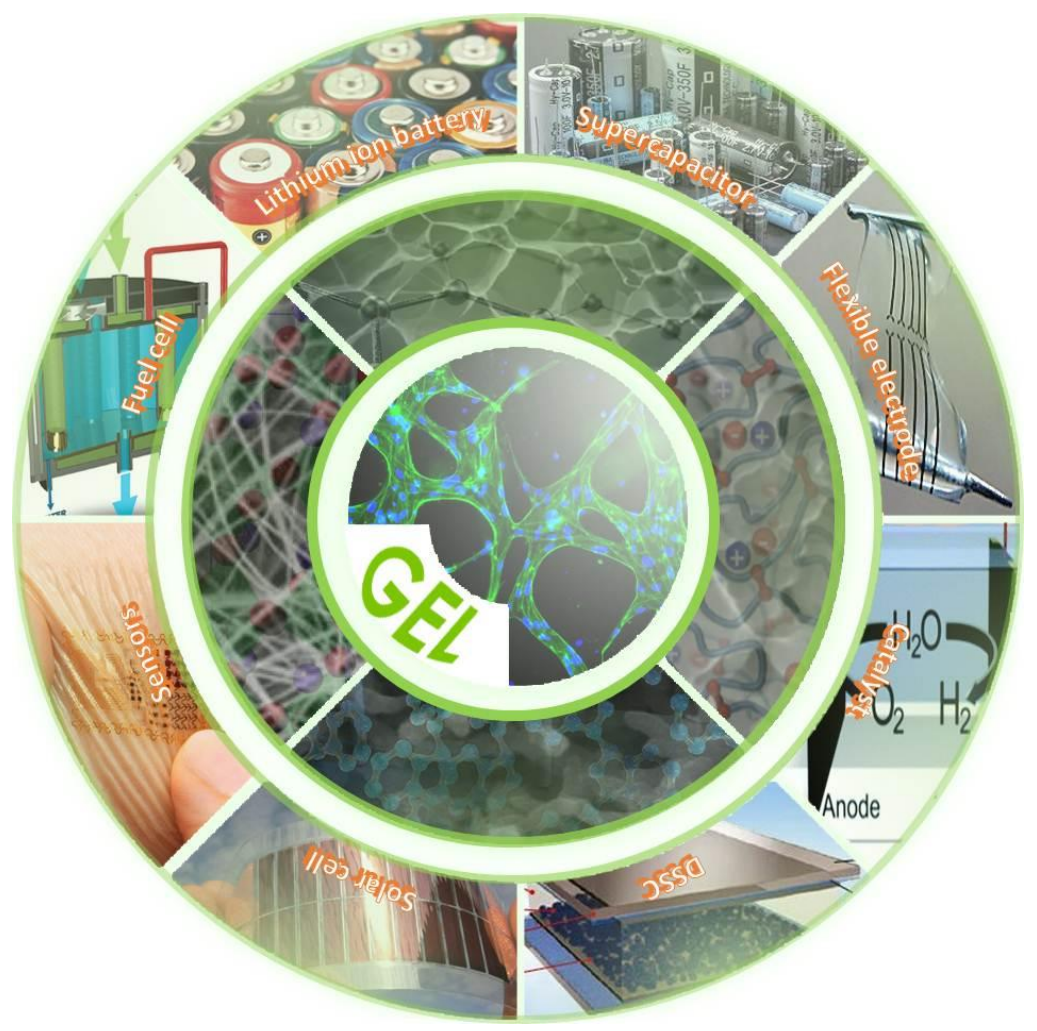

Figure 1. Four kinds of gel materials presented in this paper, including graphene-based gels, conductive polymer gels, ionically conductive gels and inorganic gels, and representative applications thereof.

\section{Carbon-based gels for energy applications}


Various carbon-based nanomaterials, including carbon nanotubes (CNTs), carbon fibers and graphene, have been successfully assembled into gel materials with 3D networks, and several comprehensive review papers have discussed these carbon-based gel materials.[8] CNTs were a typical class of carbon materials used for building up 3D structures. CNTs based gels can be prepared from CNT suspensions which are pre-prepared by using chemically modified CNTs or adding surfactants through the methods including self-assembly, critical-point-drying and freeze-drying. Physical interactions between CNTs are key driving forces for the gel formation.[8] For example, Yodh et al.[20] prepared CNTs aqueous suspensions with the surfactant of sodium dodecylbenzene sulfonate (SDS) and obtained aerogels by critical-point-drying method. This gel can be further mechanically reinforced by adding PVA polymers to achieve higher strength and stability. Besides the solution based methods, CNTs gels can be also prepared by chemical vapor deposition (CVD) in which the density and microstructures of gels can be controlled by tuning the injection rate of carbon sources or introducing solvents. These CNTs based gels are low density, highly porous materials with large surface area, thus enabling wide range of applications such as thermal and acoustic insulating materials, catalyst support, and energy storage devices.[21]

Compared to CNTs based gels, 3D structured gels based on graphene and graphene oxide (GO) exhibit similar properties such as high electrical conductivity, high thermal conductivity and high surface area. They also possess advantages including better gelation capability, facile preparation methods and elastic character which favors electrical contact between the gel electrodes and current collector in energy generation and storage devices. So graphene and GO have been more widely researched as gel precursors, and gels derived from these materials have been widely used in energy applications. Therefore, in this review, we will focus on graphene-based gel materials.

Since the discovery of the ability to obtain graphene through the "Scotch tape" peeling process in 2004, these unique 2D single sheets of carbon atoms arranged in hexagonal networks have been intensively studied. By virtue of their tremendous 
advantages, such as high surface areas, high Young's moduli, high thermal conductivity, high electrical conductivity and excellent chemical stability, graphene-based materials have been employed in a wide variety of applications, from energy conversion and storage, catalysis, and electronics to biomedical devices and novel composites. However, the performance of graphene sheets is also limited by certain intrinsic problems, especially when they are applied in electrochemical devices.[22] For example, graphene sheets tend to re-stack during preparation, which inhibits the diffusion of electrolytes. Graphene also shows a low packing density. To overcome these disadvantages, graphene-based gel materials with 3D network structures have been developed. These structures provide hierarchical pores and high surface areas as well as high strength and conductivity at low density, which could facilitate the transport of electrons and ions in electrochemical energy conversion and storage devices. Several comprehensive review papers have presented the synthesis and general applications of graphene-based gel materials.[8, 22, 23] In this section, we will focus on the applications of such material for energy conversion and storage and discuss the recent progress achieved in the field of graphene-based gels.

\subsection{Synthesis of graphene-based gels}

\subsubsection{Self-assembly without crosslinking components}

Self-assembly is a powerful technique for building up macroscopic structures from blocks at the nanoscale or molecular level. The materials thus assembled usually inherit the unique properties of building blocks at the nanoscale while exhibiting novel functionalities because of the assembled superstructures. Graphene and GO materials with unique 2D structures are versatile building blocks for the self-assembly of functional carbon-based materials, especially 3D hydrogels or aerogels. Self-assembled graphene or GO gels show unique mechanical and electrical properties compared with similar gels synthesized using crosslinkers because they are constructed by means of relatively weak physical interactions. Various driving forces have been employed for the self-assembly of graphene-based gels, such as van der Waals forces, hydrogen bonding, $\pi-\pi$ stacking, and inclusion interactions. 
Shi et al.[24] found that graphene hydrogels could be self-assembled via a one-step hydrothermal process (Figure 2A and 2B). This self-assembly is induced by $\pi-\pi$ and hydrophobic interactions between graphene sheets during hydrothermal reduction. Unlike either small molecules or macromolecules, graphene sheets with large conjugated structures can provide large numbers of $\pi-\pi$ stacking sites between which strong bonds can form, inducing the partial overlapping or coalescing of the flexible graphene sheets and thereby endowing the resulting graphene hydrogel with beneficial mechanical and electrical properties. Such a hydrogel with a water content of $\sim 97 \%$ can exhibit a compressive elastic modulus of $\sim 300 \mathrm{kPa}$ and a conductivity of

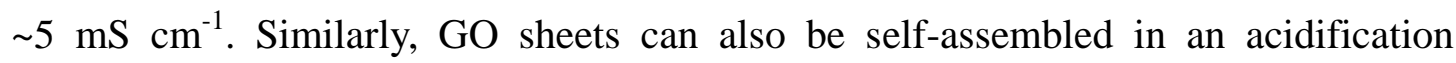
process via functional groups on the sheets.[25] The acidification of a GO dispersion weakens the electrostatic repulsion and leads to the formation of a graphite oxide flocculent. In addition, a large lateral dimension for such GO sheets greatly enhances their gelation ability. Using a simple centrifugal evaporation process, Seo et al.[26] successfully synthesized a GO gel. During the centrifugal evaporation process, GO sheets in aqueous suspensions can be self-assembled to generate network-linked aerogels or multilayered films by controlling the temperature of the vacuum chamber. 


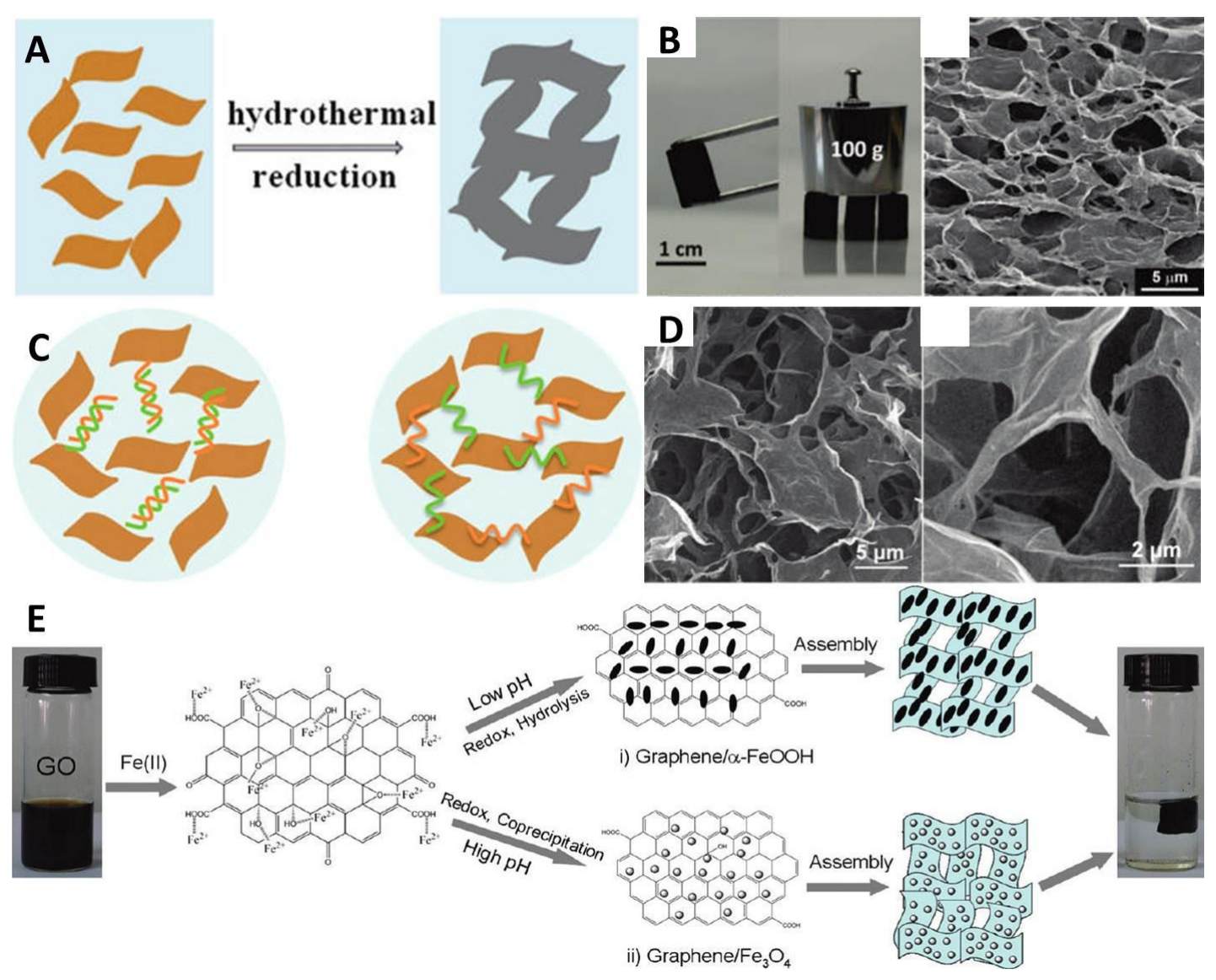

Figure 2. (A) and (B) A graphene hydrogel with a 3D networking structure self-assembled via a one-step hydrothermal process. Reproduced with permission from ref. 24. Copyright 2010 American Chemical Society. (C) and (D) A multifunctional hydrogel assembled by mixing GO and double-stranded DNA in an aqueous dispersion. Reproduced with permission from ref. 29. Copyright 2010 American Chemical Society. (E) Macroscopic multifunctional graphene-based hydrogels assembled with ferrous ions. Reproduced with permission from ref. 32. Copyright 2012 American Chemical Society.

\subsubsection{Assembly with crosslinking components}

The assembly of graphene or GO sheets can be triggered by the addition into their suspensions of a second component that provides various interactions. After the addition of gelation components such as polymers, small molecules, metal ions, or biomolecules, these entities become chemical or physical crosslinkers that ultimately drive the formation of a $3 \mathrm{D}$ structure and endow the resulting material with some 
functionality.

Polymers are some of the most commonly used gelators for the synthesis of graphene-based gels. Polymers such as polyvinyl alcohol (PVA), poly(ethylene oxide) (PEO), hydroxypropyl cellulose (HPC), and poly(vinyl pyrrolidone) (PVP) have been successfully demonstrated to induce the gelation of GO.[25] Only small amounts of these polymers are needed for gelation because they can act as either hydrogen-bonding donors or acceptors, thus providing an additional route for hydrogen-bonding interactions with adjacent GO sheets. The addition of crosslinking polymers can increase the interplanar distance of GO sheets, thereby preventing their stacking and improving the electrochemical performance of the resulting material. More importantly, this approach provides the possibility of tuning the mechanical and electrical properties of graphene gels by controlling the molecular weight of the polymer crosslinkers, because a longer polymer chain can form more hydrogen bonds with GO sheets and can interact with more than one GO sheet. In addition to hydrogen bonding, electrostatic interaction is another driving force of gelation that can be provided by polymer crosslinkers. Polydimethyldiallylammonium chloride (PDDA) and polyethylenimine (PEI) with protonated amino groups have been used to gelate GO.[25] Because of the long range and high strength of their electrostatic interactions, only low amounts of polymer crosslinkers are needed for the formation of graphene gels, thus leading to only minor negative effects on the properties of the graphene.

Small molecules can help to stabilize a graphene suspension through surface modification, in addition to introducing supramolecular interactions to drive the formation of graphene networks. Banerjee and colleagues[27] successfully synthesized GO-based supramolecular hydrogels from synthetic dipeptides, amino acids and nucleosides, which acted as physical cross-linking agents in the hydrogels. Han et al.[28] designed a series of amphiphilic molecules that possessed a polar carbohydrate head group attached to a nonpolar pyrene group and used them to construct GO gel networks via self-assembly. By tuning the molecular structure of the gelators, gelation can be achieved in both aqueous and organic solutions. The $\pi-\pi$ 
stacking interaction between pyrene groups and the surface of GO, as well as between the hydrogen bonds among the $-\mathrm{OH}$ and $-\mathrm{CO}-\mathrm{NH}-$ groups in the gelator molecules and the $-\mathrm{OH}$ and $-\mathrm{COOH}$ groups on the $\mathrm{GO}$ surface, contribute to the self-assembly of GO sheets, thereby strengthening the gel and leading to reinforced supramolecular architectures.

Biomolecules are another important class of crosslinking materials for the gelation of graphene-based gels. Shi et al.[29] reported a multifunctional hydrogel synthesized by mixing GO and double-stranded DNA in an aqueous dispersion, followed by a heating process during which the double-stranded DNA was unwound into single-stranded DNA and formed bridges adjacent GO sheets via strong non-covalent interactions (Figure 2C and 2D). A self-assembled hydrogel network was ultimately formed by means of these physical crosslinking effects and partial coalescing of the flexible GO sheets through DNA binding. Interestingly, this GO/DNA hydrogel is stable under a variety of harsh conditions, such as strongly acidic ( $\mathrm{pH} 2)$, basic ( $\mathrm{pH} 13)$, and salty $(1 \mathrm{M} \mathrm{NaCl})$ aqueous solutions, and even in organic solvents. This excellent environmental stability can be attributed to the multiple non-covalent interactions between the GO sheets and the DNA molecules, including $\pi-\pi$ stacking effects, hydrophobic interactions, and electrostatic/hydrogen bonding interactions between the prime amines of the DNA bases and the oxygen-containing groups of the GO. The same group also synthesized a $\mathrm{GO} /$ hemoglobin hydrogel by directly mixing dispersions of the two components. [30] This composite hydrogel also shows enhanced stability in organic solvents because the hydrogel network provides an aqueous microenvironment to protect the enzyme.

It has been reported that metal ions can be used to assemble GO sheets into 3D networks. The main driving force for the assembly behavior is believed to be the coordination of the metal ions with the hydroxyl and carboxyl groups on the GO sheets. Various metal ions can react with graphene sheets, but only divalent and trivalent ions such as $\mathrm{Ca}^{2+}, \mathrm{Mg}^{2+}, \mathrm{Cu}^{2+}, \mathrm{Pb}^{2+}, \mathrm{Cr}^{3+}$, and $\mathrm{Fe}^{3+}$ can act as gelators to induce the gelation of graphene.[25, 31] Multivalent transition-metal ions usually exhibit higher coordination stability and thus demonstrate better crosslinking abilities. 
As a specific example, Yu et al.[32] reported the synthesis of macroscopic multifunctional graphene-based hydrogels under the synergistic effects of the reduction of GO sheets by ferrous ions and the deposition of functional nanomaterials such as $\mathrm{FeOOH}$ nanorods and $\mathrm{Fe}_{3} \mathrm{O}_{4}$ nanoparticles onto the graphene networks (Figure 2E and 2F). Ferrous $\mathrm{Fe}^{2+}$ ions diffuse toward the GO sheets via electrostatic interactions and are oxidized into ferric ions by the oxygen-containing functional groups on the $\mathrm{GO}$ surface. At different $\mathrm{pH}$ values, either $\mathrm{FeOOH}$ or $\mathrm{Fe}_{3} \mathrm{O}_{4}$ particles will form and stabilize the GO hydrogel by means of the coordination effect. Hydrophobic and $\pi-\pi$ stacking interactions, which are strengthened by the decrease in the number of oxygenated groups on the GO sheets during reduction, also drive the assembly of the GO sheets.

\subsubsection{Template synthesis}

Template-guided synthesis is a general method of growing gels based on graphene or other carbon materials. Graphene or GO sheets can be directly deposited or grown on pre-formed backbones, resulting in interconnected graphene network structures. The advantages of template-guided methods lie in their universality and dimensional control capabilities. Various templates have been used, and the structures of the final graphene gels can be controlled by tuning the synthesis conditions.

Yavari et al.[33] used a scaffold of porous nickel foam as a template and deposited graphene sheets onto the foam via the chemical vapor deposition (CVD) method, using $\mathrm{CH}_{4}$ as the carbon source (Figure 3A and 3B). Before the Ni template was removed, a thin layer of poly(methyl methacrylate) (PMMA) was deposited on the surface of the graphene to maintain the integrity of the graphene network. Then, the nickel scaffold was removed via chemical etching with a hot $\mathrm{HCl}$ solution, and the PMMA layer was dissolved with hot acetone. The graphene gel thus obtained inherited the continuous $3 \mathrm{D}$ network structure of the $\mathrm{Ni}$ foam and possessed a free-standing macroscopic structure consisting of extremely thin interconnected sheets. The reason for this outcome is that the graphene sheets were homogeneously grown over the entire surface of the nickel foam scaffold and hence were interconnected with 
each other, with no physical breaks in the network.

Graphene-based 3D gel structures can be also prepared via the simple impregnation of templates with graphene sheets. Cui et al.[34] fabricated graphene-sponge electrodes by coating graphene onto polyurethane sponges (Figure 3C and 3D). An aqueous solution of graphene nanopowder was first prepared via sonication. Then, synthetic polyurethane sponges were dipped into the graphene dispersion, removed, and dried. This fabrication procedure is simple and scalable, and the conductive coating of graphene sheets can conform to the sponge morphology without affecting its open porous structure. In another work, Tai and colleagues[35] fabricated graphene-based sponges via a facile and inexpensive dip-coating method using commercially available melamine sponges as the templates. A melamine sponge was dipped into the graphene/ethanol dispersion and then dried in a vacuum oven. The mechanical flexibility of graphene nanosheets and the strong van der Waals interactions ensured the physical linking of the graphene nanosheets onto the sponge skeletons while maintaining the 3D network structure.
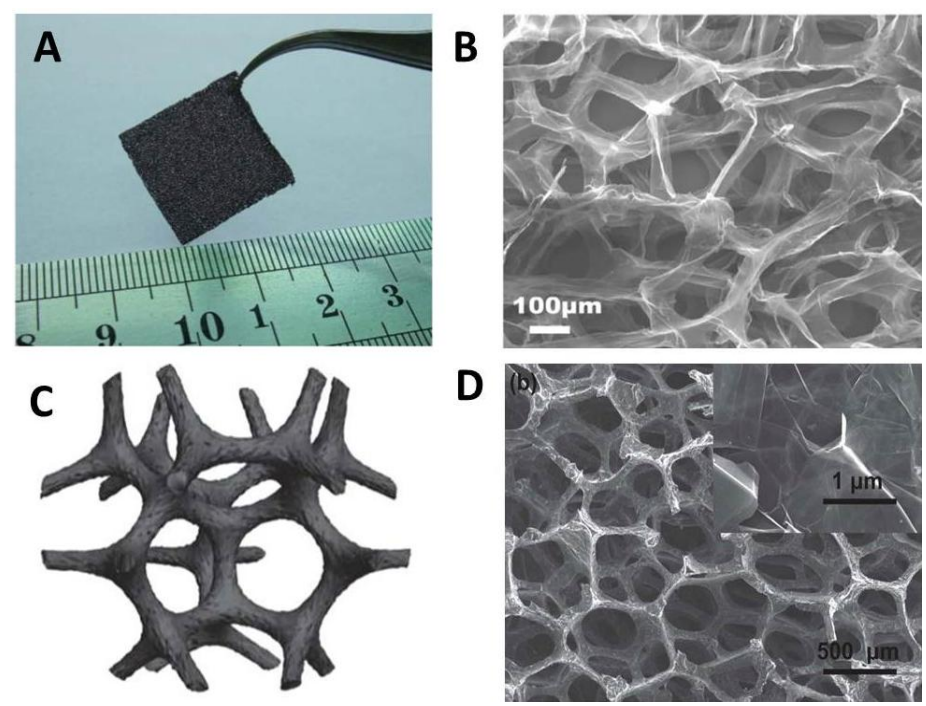

Figure 3. (A) and (B) A scaffold of graphene sheets deposited via CVD using porous nickel foam as the template. Reproduced with permission from ref. 33. Copyright 2011 Nature Publishing Group. (C) and (D) A graphene-sponge electrode fabricated by coating graphene onto a polyurethane sponge. Reproduced with permission from ref. 34. Copyright 2012 The Royal Society of Chemistry. 


\subsection{Energy applications of graphene-based gels}

\subsubsection{Supercapacitors}

Supercapacitors are regarded as an important class of electrochemical energy storage devices because of their high power densities, long life cycles and potential to reach relatively high energy densities. Carbon materials such as graphite, carbon nanotubes, and activated porous carbon have been used as electrode materials for supercapacitors because of their ability to accumulate charges in the electric double layer that forms at every electrode/electrolyte interface. The specific capacitance of a carbon material strongly depends on its surface area, electrical conductivity and ability to facilitate the flow of electrolyte. As a unique carbon nanomaterial, graphene can fundamentally provide a specific capacitance of up to $550 \mathrm{~F} \mathrm{~g}^{-1}$, primarily by virtue of its excellent conductivity, high mechanical strength and flexibility, large specific surface area, and chemical stability.[36] However, graphene sheets tend to re-stack when processed into bulk electrodes because of strong $\pi$ - $\pi$ stacking, which leads to a decrease in the active surface area and the inhibition of ionic diffusion. The gelation of graphene into a 3D network structure can suppress this re-stacking, and the resulting porous structure can provide multidimensional electron transport pathways, easy access to the electrolyte, and minimal transport distances between the bulk of the electrode and the electrolyte, thereby achieving an enhanced capacitance.

Shi et al.[36] synthesized a self-assembled graphene hydrogel via a one-step hydrothermal process followed by a chemical reduction process using hydrazine or hydroiodic acid as a reduction agent. A supercapacitor fabricated based on this $3 \mathrm{D}$ porous material had a high specific capacitance of $220 \mathrm{~F} \mathrm{~g}^{-1}$ at a current density of $1 \mathrm{~A}$ $\mathrm{g}^{-1}$ and could maintain a high specific capacitance of $165 \mathrm{~F} \mathrm{~g}^{-1}$ at an ultrafast discharge rate of $100 \mathrm{~A} \mathrm{~g}^{-1}$ in aqueous electrolyte. This superior performance was attributed to the modified chemical structure and morphology of the graphene hydrogel. The chemical reduction removed residual oxygenated groups from the graphene sheets and further improved their conductivity. The unique 3D morphology allowed most of the graphene sheets to be exposed to the electrolyte and provided open channels to facilitate the transport of ions. It is also worth noting that this 
graphene-hydrogel-based electrode was fabricated without any conductive additives or polymer binder, thus simplifying the processing and eliminating the disadvantageous effects of additional agents. Because of the high mechanical strength and flexibility of graphene hydrogels, flexible solid-state supercapacitors can be fabricated using such materials. Duan et al.[37] developed a solid-state supercapacitor using graphene hydrogel films as the electrodes and $\mathrm{H}_{2} \mathrm{SO}_{4}$-polyvinyl alcohol (PVA) gel as the electrolyte (Figure 4A to 4C). This device, with a $120-\mu \mathrm{m}$-thick graphene hydrogel thin film, exhibited a high gravimetric specific capacitance of $186 \mathrm{~F} \mathrm{~g}^{-1}$, an unprecedented areal specific capacitance of $372 \mathrm{mF} \mathrm{cm}^{-2}$, excellent cycling stability, and extraordinary mechanical flexibility.

Various strategies have been developed to further enhance the electrochemical performance of graphene-based gel materials as supercapacitor electrodes. The surfaces of the graphene sheets in a gel matrix can be modified for this purpose. Duan et al.[38] functionalized graphene hydrogels through a one-step chemical reduction of GO using hydroquinones as the reducing and functionalizing molecules simultaneously (Figure 4D to 4F). By virtue of the large surface area of these graphene hydrogels, they could accommodate a large number of hydroquinone molecules, leading to a greatly enhanced contribution to the pseudocapacitance. In addition, all of the hydroquinone molecules were directly attached to the graphene sheets via $\pi-\pi$ interactions, which allowed full use to be made of the pseudocapacitive component and afforded rapid electron transfer from the graphene substrates to the hydroquinone for rapid Faradaic reactions. With these functionalized graphene hydrogels as electrodes, a supercapacitor with a specific capacitance of $441 \mathrm{~F} \mathrm{~g}^{-1}$ was achieved. To enhance the surface area, the same group synthesized a holey graphene framework by adding $\mathrm{H}_{2} \mathrm{O}_{2}$ during the self-assembly of the graphene sheets.[39] The $\mathrm{H}_{2} \mathrm{O}_{2}$ molecules etched the carbon atoms around active defect sites of GO, leaving behind carbon vacancies, which gradually extend into nanopores across the entire sheets. The resulting holey gel framework exhibited a large specific surface area $\left(1560 \mathrm{~m}^{2} \mathrm{~g}^{-1}\right)$ and enhanced mechanical strength. More importantly, the pores were sufficiently large to enable efficient ion transport throughout the entire network, even 
in a highly compressed form. A fully packaged supercapacitor stack based on these holey graphene hydrogels was found to deliver gravimetric and volumetric energy densities of $35 \mathrm{Wh} \mathrm{kg}^{-1}$ and $49 \mathrm{Wh} \mathrm{L}^{-1}$, respectively, approaching the corresponding value for lead acid batteries.

Another method of developing high-performance electrode materials is to dope the frameworks of graphene gels with nitrogen, boron, sulfur and other atoms. Yu et al.[40] synthesized nitrogen-doped graphene hydrogels using organic amines and GO as precursors (Figure 4G to 4I). By using different species and amounts of the organic amines, the microstructural parameters of the graphene hydrogels, such as the pore size, could be tuned. $\mathrm{N}$ doping in the carbon plane could expand the separation and hinder the $\pi-\pi$ stacking between graphene sheets because of the dangling chains of the organic amines outside the plane and the formation of hydrogen bonds, thereby improving the electrochemical performance. The resulting supercapacitors could reach a high power density of $205.0 \mathrm{~kW} \mathrm{~kg}^{-1}$ at an ultrafast charge/discharge rate of 185.0 $\mathrm{A} \mathrm{g}^{-1}$. In another study, Feng at al.[41] co-doped graphene aerogels with nitrogen and boron using ammonia boron trifluoride $\left(\mathrm{NH}_{3} \mathrm{BF}_{3}\right)$ as the precursor via a hydrothermal reaction. The electrical conductivity of the graphene framework was enhanced, and a high power density of $\sim 1600 \mathrm{~W} \mathrm{~kg}^{-1}$ was achieved.

In addition to chemical modification, novel techniques for the microstructural engineering of graphene gels and for device structure design have been proposed to better exploit the potential of graphene-based gels for electrochemical applications. Kim et al.[42] developed a simple and versatile graphene gelation method capable of the shape engineering of micrometer-thick hydrogels based on the immersion of arbitrarily shaped $\mathrm{Zn}$ objects in an aqueous GO dispersion. Hydrogel films were spontaneously generated at the Zn surfaces, and this site-specific gelation enabled a wide range of controllability because the thickness could be tuned by varying the immersion time and the area could be tuned by varying the $\mathrm{Zn}$ film size. The resulting graphene-gel-based supercapacitors exhibited an energy density as high as $2.73 \mu \mathrm{Wh}$ $\mathrm{cm}^{-2}$ and a high areal power density of $369.8 \mathrm{~mW} \mathrm{~cm}^{-2}$. Beyond simply symmetrically structured supercapacitors, graphene hydrogels have been also used to fabricate 
asymmetric supercapacitors to achieve higher performance. Duan et al.[43] fabricated an asymmetric supercapacitor using a graphene hydrogel as the negative electrode and vertically aligned $\mathrm{MnO}_{2}$ nanoplates on nickel foam as the positive electrode. The two electrodes exhibited complementary potential windows. The supercapacitor thus obtained could be cycled through a wide potential range of $0-2.0 \mathrm{~V}$ and exhibited an energy density of $23.2 \mathrm{Wh} \mathrm{kg}^{-1}$ with a power density of $1.0 \mathrm{~kW} \mathrm{~kg}^{-1}$.
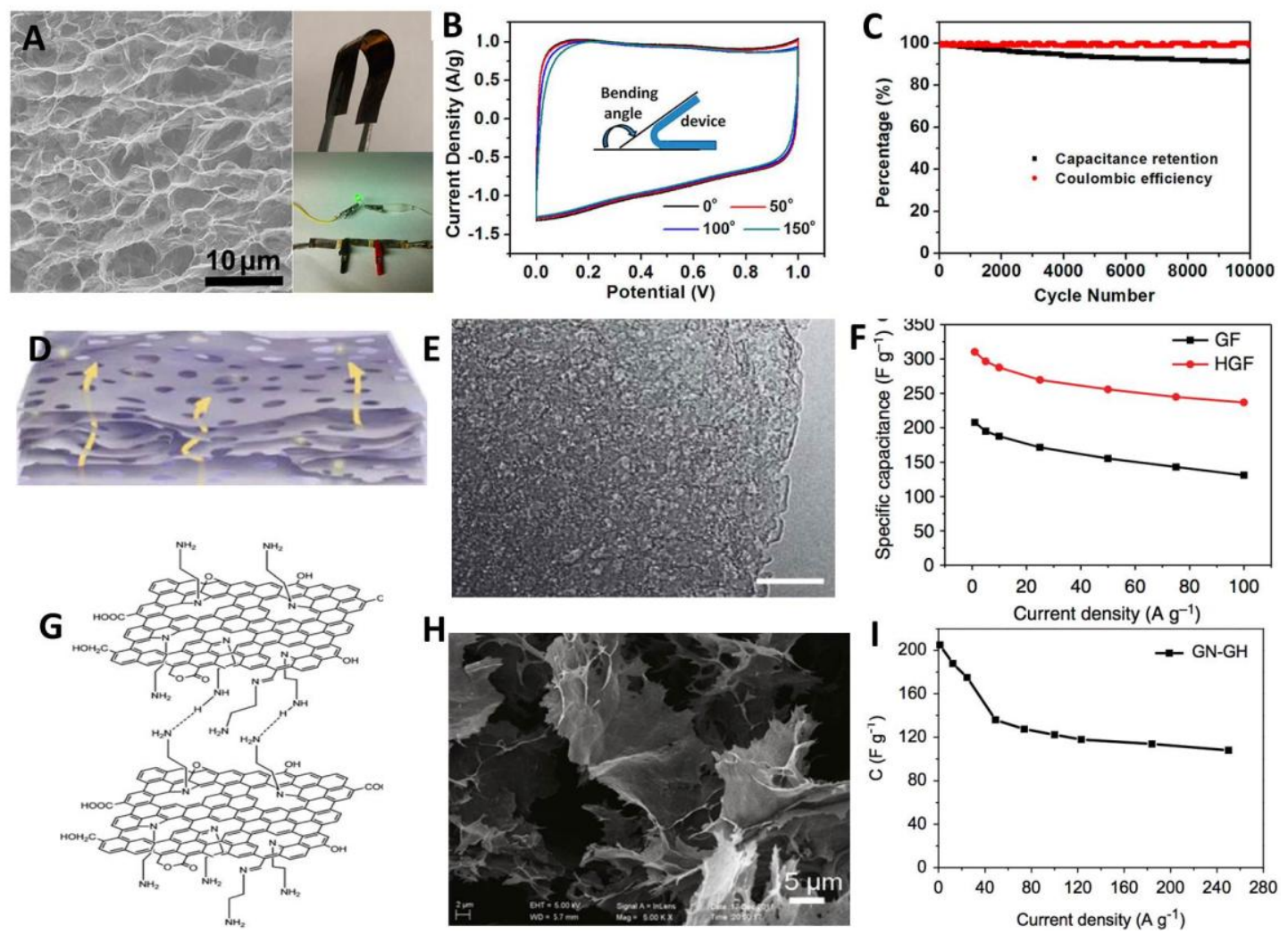

Figure 4. (A) to C) A solid-state supercapacitor with graphene hydrogel films as the electrodes and $\mathrm{H}_{2} \mathrm{SO}_{4}$-polyvinyl alcohol (PVA) gel as the electrolyte. (B) and (C) $\mathrm{CV}$ and cycling performance of the solid-state supercapacitor. Reproduced with permission from ref. 37. Copyright 2013 American Chemical Society. (D) A schematic illustration of a holey graphene gel framework and the corresponding (E) TEM image and (F) electrochemical performance. Reproduced with permission from ref. 38. Copyright 2013 WILEY-VCH Verlag GmbH \& Co. (G to I) A nitrogen-doped graphene hydrogel obtained using organic amines and GO as precursors and its electrochemical performance. Reproduced with permission from ref. 40. Copyright 2013 Elsevier Ltd. 


\subsubsection{Lithium-ion batteries}

Lithium-ion batteries (LIBs) are widely used in consumer electronics because of their high energy density. Graphene has been demonstrated to be a promising anode material for LIBs because of its high electrical conductivity, mechanical robustness and large surface area. The theoretical energy density of graphene is $372 \mathrm{mAh} \mathrm{g}^{-1}$, when single-sided $\mathrm{Li}$ adsorption occurs to form $\mathrm{LiC}_{6}$, or $744 \mathrm{mAh} \mathrm{g}^{-1}$, when $\mathrm{Li}$ ions are adsorbed on both sides of the graphene.[44] However, graphene faces the problem of aggregation due to its large surface area and the strong van der Waals interactions between sheets during electrode preparation. As a result, the cross-plane diffusivity of $\mathrm{Li}$ ions is low, and the migration of $\mathrm{Li}^{+}$ions into and out of a graphene anode may occur predominantly at the edge of the stack. This greatly limits the electrochemical performance of such an electrode, particularly at high charge/discharge rates.

It is desirable to construct 3D structured graphene gels to improve the diffusion of $\mathrm{Li}^{+}$into the interspaces between the graphene sheets, thereby enhancing the electrochemical utilization of the graphene. Using this strategy, Manthiram et al.[45] synthesized a stacked porous graphene structure, which was subsequently etched with $\mathrm{KOH}$ to form holes (Figure $\mathbf{5 A}$ to $\mathbf{5 C}$ ). The resulting material showed a high rate capability with excellent cycling stability when used as an anode material for LIBs. At a high rate of $10 \mathrm{C}$, such an electrode can still deliver a reversible specific capacity of $147 \mathrm{mAh} \mathrm{g}^{-1}$. An EIS study revealed that the diffusion coefficient for Li ions could reach $\sim 1.4 \times 10^{-8} \mathrm{~cm}^{2} \mathrm{~s}^{-1}$, which is comparable to the reported Li-ion diffusivity in graphite and thus supports the claim of high performance. In another study, Wang et al.[44] prepared graphene hydrogels with carbon nanotubes as the crosslinkers and applied the obtained material as anodes in LIBs. The material exhibited a high initial reversible specific capacity of $439 \mathrm{mAh} \mathrm{g}^{-1}$ at a current density of $372 \mathrm{~mA} \mathrm{~g}^{-1}$, and a high capacity of $429 \mathrm{mAh} \mathrm{g}^{-1}$ was maintained after 100 cycles.

In addition to being directly used as anode materials in LIBs, graphene gels are also commonly used as frameworks to support active materials. In this case, the battery performance is determined not only by the intrinsic properties of the active 
material but also by the electrode properties and structures, such as the electronic/ionic conductivity and the pore structure. A graphene gel can provide a 3D network with a high electrical conductivity, thereby increasing the electron transfer rate in the electrode. The large surface area can support a large quantity of active materials and increase the interfacial area, thereby increasing the number of active sites for electrochemical reactions. The 3D pore network enables rapid $\mathrm{Li}^{+}$transport. In addition, for active materials such as transition-metal oxides and sulfides that exhibit large specific volume changes during cycling, which can lead to pulverization of the electrodes and aggregation of the electrode materials, graphene gels can efficiently accommodate these volume changes.

As a candidate cathode material, $\mathrm{LiFePO}_{4}$ demonstrates various advantages, such as a high theoretical capacity $\left(170 \mathrm{mAh} \mathrm{g}^{-1}\right)$, high electrochemical potential, good thermal stability, a low cost, environmental friendliness, and nontoxicity. However, its performance is also limited by poor electrical conductivity and an intrinsically low Li-ion diffusivity. Wang et al.[46] wrapped $\mathrm{LiFePO}_{4}$ nanoplatelets in a nitrogen-doped graphene gel using a self-assembly method in which the electrostatic attraction between the positively charged aminopropyltrimethoxysilane (APS)-modified $\mathrm{LiFePO}_{4}$ nanoplatelets and the negatively charged GO acted as the driving force of gelation (Figure 5D and 5E). The resulting hybrid electrode achieved a high rate capability (78 $\mathrm{mAh} \mathrm{g}^{-1}$ at $100 \mathrm{C}$ ) and a long life-cycle stability (89\% capacity retention over 1000 cycles at $10 \mathrm{C}$ ). The mechanism is that the nitrogen-doped graphene gel with its interconnected pore network provides pathways for rapid electron transfer and ion transport.

Similar strategies have also been used to develop anode materials for LIBs. Ajayan et al.[47] synthesized 3D graphene-backboned hybrid architectures with $\mathrm{MoS}_{2}$ and $\mathrm{FeO}_{\mathrm{x}}($ Figure $\mathbf{5 F}$ to $\mathbf{5 H})$. The graphene network backbone not only improved the conductivity of the overall electrode but also strongly enhanced the electrochemical activity of the active materials during cycling. As a result, a long cycle life was achieved (3000 cycles for $\mathrm{MoS}_{2}$ and 1500 cycles for $\mathrm{FeO}_{\mathrm{x}}$ with nearly $100 \%$ capacity retention) at different charge and discharge rates. Moreover, the rate capabilities for 
$\mathrm{MoS}_{2}$ /graphene and $\mathrm{FeO}_{\mathrm{x}}$ /graphene were up to $140 \mathrm{C}$ and $80 \mathrm{C}$, respectively. $\mathrm{Si}$ nanoparticles were also introduced into a graphene gel framework by Chen et al.[48] The hybrid electrode material exhibited a greatly improved cycle stability (85\% capacity retention after 200 cycles at $1 \mathrm{~A} \mathrm{~g}^{-1}$ ), a high specific capacity, and good rate performance.

A porous graphene gel can also enhance the mass loading of active materials. To date, Li-S batteries have not been commercialized because most as-prepared electrodes for such batteries have extremely low areal mass loadings, causing the real capacity of these battery systems to be greatly decreased compared with that of commercial LIBs. The use of a graphene gel matrix is a promising way of solving this problem. Wu et al.[49] reported an approach in which self-supported graphene sponges were first obtained via hydrothermal reduction and sulfur was then impregnated into the pores of the as-prepared graphene sponges through a heat treatment. The resulting porous graphene sponges not only exhibited improved overall conductance when used as cathodes but also absorbed the intermediate soluble polysulfides. This composite material could be directly used as a cathode with a large areal mass loading of sulfur $\left(12 \mathrm{mg} \mathrm{cm}^{-2}\right)$, showing a high areal specific capacity of $4.53 \mathrm{mAh} \mathrm{cm}^{-2}$ and good stability ( $0.08 \%$ decay per cycle after 300 cycles). Graphene aerogels with 3D porous structures can also be used as support structures for sulfur to enhance the conductivity of electrodes and to suppress the dissolution of intermediate products during charging/discharging.[50] 

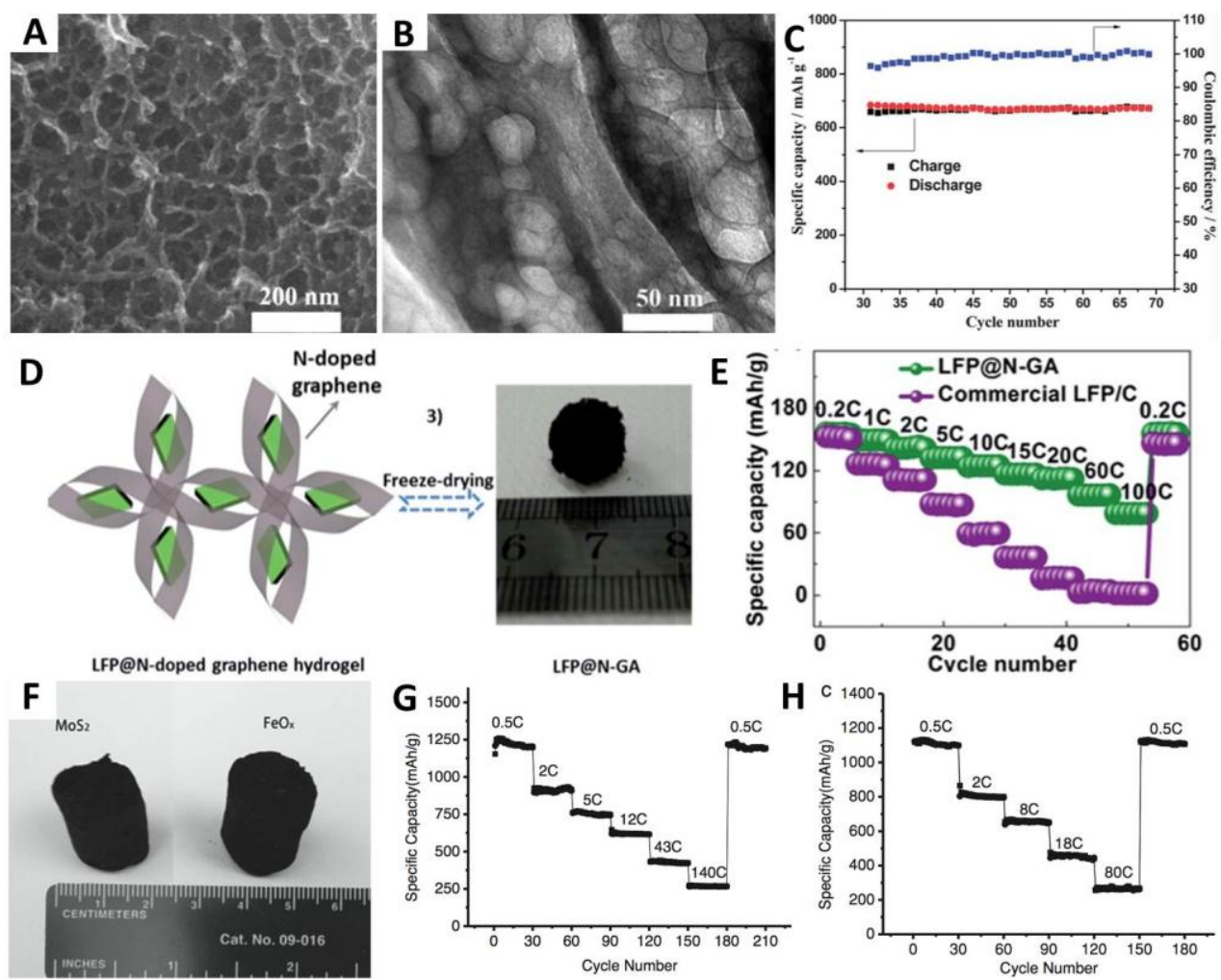

Figure 5. (A to C) SEM and TEM images of a stacked porous structure of graphene with holes formed through $\mathrm{KOH}$ etching, showing a high rate capability and excellent cycling stability when used as an anode material for LIBs. Reproduced with permission from ref. 45. Copyright 2013 Royal Society of Chemistry. (D) Schematic illustration and photograph of $\mathrm{LiFePO}_{4}$ nanoplatelets wrapped in a nitrogen-doped graphene gel produced via a self-assembly method. (E) Rate performance of the gel-wrapped $\mathrm{LiFePO}_{4}$ nanoplatelets. Reproduced with permission from ref. 46. Copyright 2015 Royal Society of Chemistry. (F to H) 3D graphene-backboned hybrid architectures with $\mathrm{MoS}_{2}$ and $\mathrm{FeO}_{\mathrm{x}}$ and their rate performance for lithium ion battery. Reproduced with permission from ref. 47. Copyright 2013 WILEY-VCH Verlag GmbH \& Co.

\subsubsection{Energy conversion}

Energy-related electrochemical reactions such as the hydrogen evolution reaction (HER), the oxygen evolution reaction (OER), and the oxygen reduction reaction (ORR) are basic reactions that occur in various energy conversion and storage systems, such as solar cells, fuel cells, and metal-air batteries. Noble metals such as Pt, 
$\mathrm{Ru}$ and Ir have been demonstrated to show high catalytic performance toward these reactions, but their large-scale applications are limited because of their scarcity and prohibitive cost. Recently, graphene and graphene-based hybrid catalysts have emerged as promising candidates for electrochemical reactions by virtue of their high catalytic activity, relatively low cost and noble-metal-free nature. The adoption of graphene-based gel materials can lead to further improvement in catalytic performance because of their unique structural and chemical properties. These self-supported gel materials exhibit good mechanical properties and can serve directly as working electrodes, ensuring strong catalyst durability. In particular, highly hydrophilic frameworks of hydrogel-based materials can yield electrodes with greatly increased wettability, facilitate access to the electrolyte, and consequently enhance the activity and reaction kinetics. In addition, the pores of the gel matrix can permit facile gas transport within electrodes.

Several studies have successfully demonstrated the potential of graphene-gel-based catalysts. Qiao et al.[51] prepared a N,O-dual-doped graphene-CNT hydrogel electrocatalyst via the assembly of chemically converted graphene (with intrinsic oxygen impurities) and CNTs through a simple filtration procedure followed by $\mathrm{N}$ doping with ammonia (Figure 6A and 6B). This self-supported material showed higher OER activity than that of a noble metal catalyst $\left(\mathrm{IrO}_{2}\right)$ and some transition-metal catalysts as well as robust stability in both alkaline and strongly acidic solutions. The catalytic kinetics was significantly enhanced because of the excellent structural properties of the material, such as its 3D framework, well-developed porosity and co-doping with $\mathrm{N}$ and $\mathrm{O}$, which provided numerous catalytic centers $(\mathrm{C}-\mathrm{N}$ and $\mathrm{C}-\mathrm{O}-\mathrm{C})$ for the OER.

Graphene-gel-based composites with other active particles have also been developed as hybrid catalysts for electrochemical reactions. Qiao et al.[52] synthesized NiCo- and $\mathrm{MoS}_{\mathrm{x}}$-decorated N-doped graphene hydrogels for the OER and HER, respectively. These hybrid materials combined a number of excellent structural properties for electrocatalysis, such as the presence of highly active sites from the doping with active species, good mechanical properties, a highly hydrated framework 
and the existence of a 3D conductive network (Figure 6C and 6D). Significant enhancements of catalytic activity and stability were demonstrated for these hybrid catalysts. The NiCo-graphene hydrogel catalyst exhibited a low overpotential of 350 $\mathrm{mV}$ and a high catalytic current density of $145.3 \mathrm{~mA} \mathrm{~cm}^{-2}$ toward the OER, whereas the MoS-graphene hydrogel catalyst demonstrated catalytic activity with an overpotential of $140.6 \mathrm{mV}$ toward the HER. In another study, an N-doped graphene aerogel with $\mathrm{Fe}_{3} \mathrm{O}_{4}$ nanoparticles was demonstrated to be an efficient cathode catalyst for the ORR.[53] Compared with $\mathrm{Fe}_{3} \mathrm{O}_{4}$ NPs supported on N-doped carbon black or $\mathrm{N}$-doped graphene sheets, this gel-based material showed a more positive onset potential, a higher cathodic density, a lower $\mathrm{H}_{2} \mathrm{O}_{2}$ yield, and a higher electron transfer number for the ORR in alkaline media, thus highlighting the importance of the $3 \mathrm{D}$ porous structure and high specific surface area of the graphene gel support in achieving improved ORR performance.
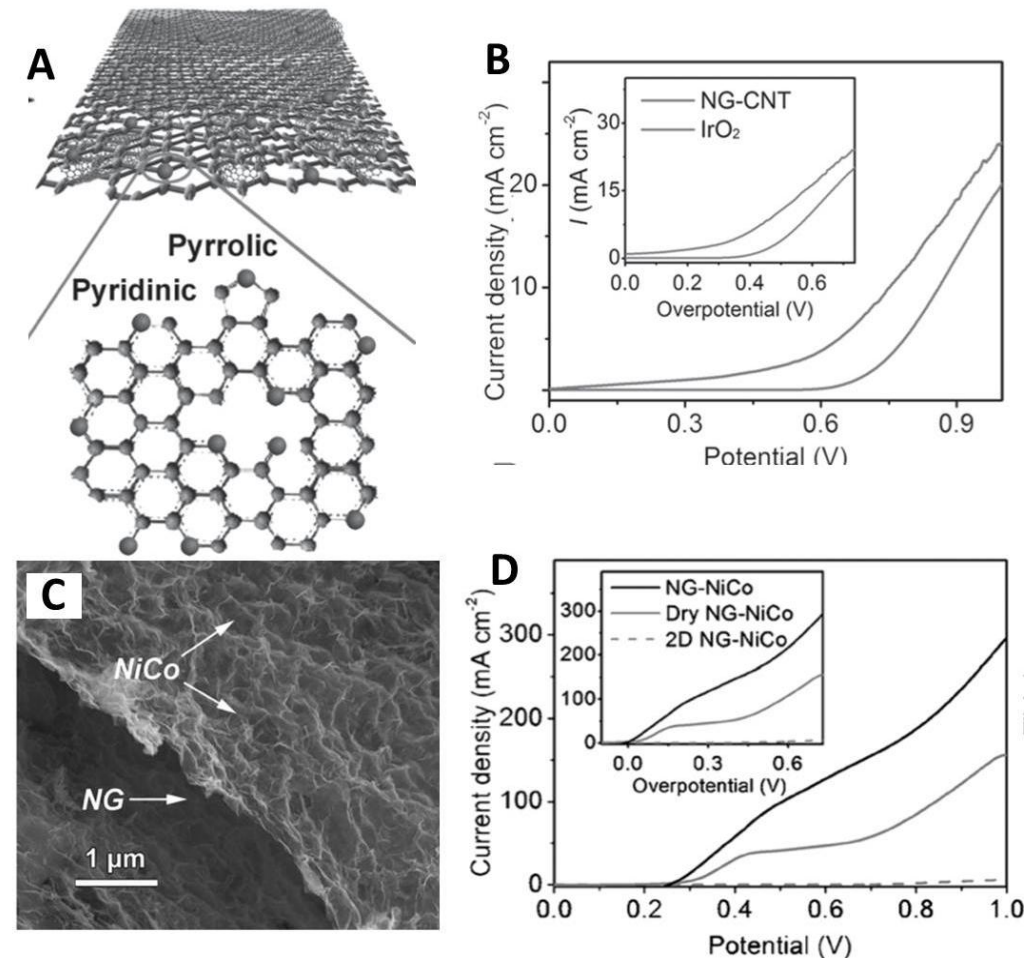

Figure 6. (A) and (B) An N,O-dual-doped graphene-CNT hydrogel electrocatalyst for the OER prepared via the assembly of chemically converted graphene and CNTs. Reproduced with permission from ref. 51. Copyright 2014 WILEY-VCH Verlag $\mathrm{GmbH} \&$ Co. (C) and (D) NiCo-decorated N-doped graphene hydrogel catalyst for the OER. Reproduced with permission from ref. 52. Copyright 2015 Elsevier Ltd. 


\subsection{Perspective}

Graphene-based gels are some of the most promising candidates to serve as next-generation materials in electrochemical energy conversion and storage applications because they combine the features of single graphene sheets and 3D network structures, such as high conductivities, high surface areas, porous structures and structural and chemical tunability. However, graphene-based gels are subject to certain trade-offs when applied in electrochemical energy devices. For example, defects on graphene can promote its performance but can also reduce its conductivity, and a porous structure favors ion diffusion but also results in a low packing density. Therefore, the microstructures and chemical states of graphene-gel-based energy materials should be carefully designed and controlled. The numbers and positions of doping-induced defects, functional groups and introduced active materials must be controlled. Small-sized spacers must be developed to facilitate the construction of graphene materials with high packing densities. Fundamental studies of the charge transfer and storage mechanisms of 3D structured graphene gels could also provide a potential direction for innovations in structural design and modification. The development of graphene-based materials is a multidisciplinary field that will require the efforts of researchers from chemistry, physics, and nanotechnology as well as various areas of engineering.

\section{Conductive polymer gels for energy applications}

A variety of conductive polymers have been developed, including polyacetylene (PA), polyaniline (PANI), polypyrrole (PPy), polythiophene (PTh), and poly(phenylenevinylene) (PPV), and they have been applied in a wide range of applications, such as energy conversion and storage, flexible electronics, and bio-medical devices, because of their combined features of organic conductors and conventional polymeric materials. Unlike metals and inorganic semiconductors, a conductive polymer can transport electrons by virtue of their $\pi$-conjugated chains and a "doping" process that involves chemical or electrochemical redox reactions and 
protonation. Their conductivities are tunable via modifications to their molecular structures, the level of doping, and the ordering of the molecular packing.[54] Conductive polymers exhibit mechanical properties similar to those of conventional polymeric materials, and they show the potential to be produced in various structures through facile solution processing. Conductive polymer gels (CPGs) show several advantages when applied for energy conversion and storage:[55-57] 1. they provide an electrically conductive yet monolithic framework to promote the transport of electrons; 2. they offer both micro- and meso-scale pores within their polymeric matrices to facilitate the diffusion of ions and molecules, respectively; 3 . they possess high surface areas, which extend the effective interfaces between the molecular chains and the electrolyte for redox reactions; 4. the porous structures of CPGs can help to accommodate the strains induces by volume changes during electrochemical reactions; and 5. their advantageous mechanical properties, including strength and elasticity, offer the potential to fabricate lightweight and flexible devices.

\subsection{Synthesis of conductive polymer gels}

Conventionally, CPGs are synthesized using template-guided methods in which pre-prepared templates or in situ polymerized templates are employed. These methods have been comprehensively summarized in other review articles.[58] However, CPGs that are synthesized via template-guided methods consist of both conductive and nonconductive components, which may lead to deterioration in their electrical properties over time and limit their scalability.

To overcome the disadvantages of template-guided synthesis, a new synthesis strategy for CPGs in which molecules with multiple functional groups are used as crosslinkers has been developed (Figure 7A to 7C).[56] Each of these crosslinking molecules can interact with more than one conductive polymer chain by protonating the nitrogen groups, thus crosslinking the polymer chains into a mesh-like hydrogel network while also acting as dopants. PANI and PPy hydrogels have been successfully synthesized using crosslinking molecules such as phytic acid and copper phthalocyanine-3,4',4",4"'-tetrasulfonic acid tetrasodium salt (CuPcTs). The resulting 
CPGs exhibit both high electrical and ionic conductivities because the 3D framework for electron transport is free of insulating polymers and the pore structure facilitates the diffusion of electrolyte ions. In addition, the swelling nature of this polymeric network may provide additional interfaces between the polymer chains and the solution phase, leading to a larger number of active reaction sites.

Dopant-molecule-crosslinked CPGs also show high tunability in terms of chemical and physical properties. The microstructure of a CPG and its derived mechanical properties can be tuned by controlling the solvents and the ratio of polymer monomers to crosslinkers. For example, an interfacial synthesis using an organic solvent and water can generate an interconnected hollow-sphere microstructure (Figure 7D to 7F).[59] The microstructural parameters, such as the microsphere diameter, size dispersion and shell thickness, can be further adjusted by using different organic solvents and by varying the ratio of polymer monomers to crosslinkers, resulting in a CPG foam with greatly enhanced elasticity.[60] The microstructures of CPGs are also determined by the molecular and geometric structures of different dopant molecules.[61] A PPy gel with an interconnected fiber structure has been synthesized using CuTcPs as the crosslinker. When indigo carmine and indigo carmine dehydrate, which have molecular structures similar to one half and one quarter of the CuPcTs molecule, respectively, are used as crosslinkers, PPy gels with a 'necklace-like' 1D nanostructure and a granular nanostructure, respectively, are obtained (Figure 7G to 7I). More importantly, the electrical and electrochemical properties of CPGs are closely related to their microstructures and to the dopants used in their synthesis. 


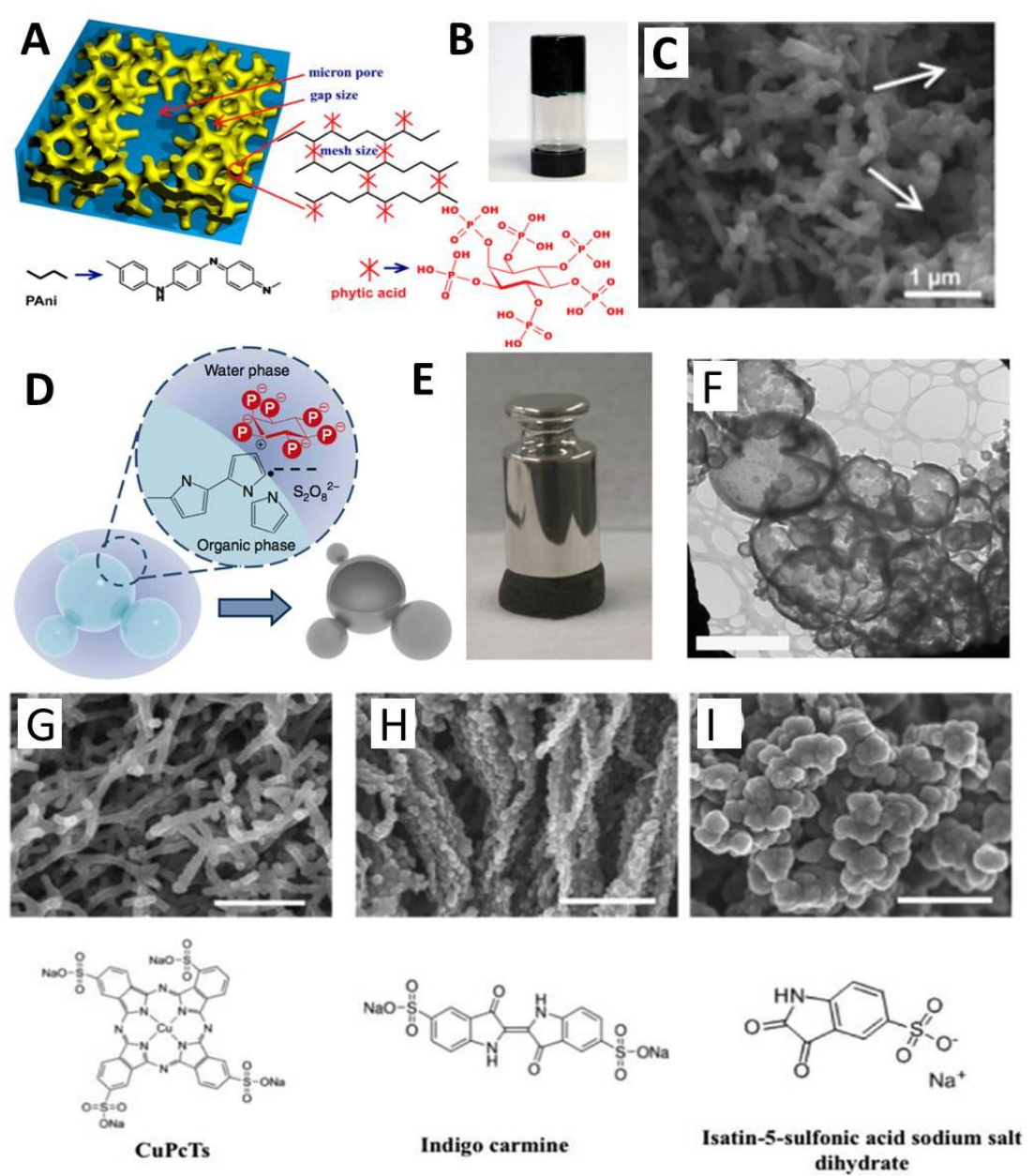

Figure 7. (A) Schematic illustration of the 3D hierarchical microstructure of a gelated PANI hydrogel obtained using phytic acid as both dopant and crosslinker. (B) A photograph of the PANI hydrogel. (C) An SEM image showing the interconnected network of dendritic PANI nanofibers. Reproduced with permission from ref. 56. Copyright 2012 Proceedings of the National Academy of Sciences of the United States of America. (D) Schematic illustration of the interfacial synthesis of hollow-sphere-structured PPy. (E) A photograph showing a spongy PPy gel sample placed under a balance weight of $100 \mathrm{~g}$. (F) TEM image of PPy showing its interconnected hollow-sphere structure, where the scale bar represents $1 \mu \mathrm{m}$. Reproduced with permission from ref. 59. Copyright 2014 Nature Publishing Group. (G-I) SEM images of different nanostructured PPy hydrogels with various dopants: (G) CuPcTs as the dopant, $(\mathrm{H})$ indigo carmine as the dopant, and (I) isatin-5-sulfonic acid sodium salt dehydrate as the dopant. The scale bar represents $1 \mu \mathrm{m}$. Reproduced with permission from ref. 61. Copyright 2015 American Chemical Society. 


\subsection{Energy applications of conductive polymer gels}

Conductive polymers are an important class of pseudocapacitive materials that take advantage of fast and reversible electron-exchange reactions at or near the electrode surface and are widely used in supercapacitors. These conjugated polymers show high gravimetric and volumetric pseudocapacitance in various non-aqueous electrolytes, and with mechanical features similar to those of conventional polymers, they are potentially useful for the fabrication of flexible and lightweight devices. However, bulk conductive polymers suffer from poor cycle lives and rapidly decaying capacitances under high-rate cycling, possibly because of the volume changes experienced during the charge/discharge processes and the decrease in conductivity caused by the change in the doping state. To solve these problems, CPGs with 3D network structures are used because they can promote the transport of electrons, provide short diffusion paths for electrolyte ions to access the electro-active surfaces, and present a porous architecture to accommodate volume changes. In addition, the doping states of CPGs are relatively stable because of the chemical bonds between the doping molecules and the polymeric chains.

Dopant-molecule-crosslinked CPGs have demonstrated excellent performance in supercapacitor applications. A PANI hydrogel was tested in a $1 \mathrm{M} \mathrm{H}_{2} \mathrm{SO}_{4}$ electrolyte and found to exhibit a specific capacitance of $\sim 480 \mathrm{~F} \mathrm{~g}^{-1}$ at a current density of $0.2 \mathrm{~A}$ $\mathrm{g}^{-1}$.[56] Because of its highly conductive framework and porous structure, the PANI hydrogel exhibited excellent rate performance, with a capacitance loss of only $~ 7 \%$ when the current density was increased by a factor of 10. A PPy hydrogel crosslinked with CuPcTs also showed a greatly enhanced specific capacitance compared with its pristine form (without any crosslinkers).[61] By virtue of its higher conductivity, 1D morphology, and more porous structure, the specific capacitance of the CuPcTs-doped PPy was calculated to be as high as $\sim 400 \mathrm{~F} \mathrm{~g}^{-1}$ at $0.2 \mathrm{~A} \mathrm{~g}^{-1}$, whereas that of the pristine PPy was only $232 \mathrm{~F} \mathrm{~g}^{-1}$.

CPGs share advantageous mechanical properties such as high elasticity and flexibility with conventional polymeric materials, and these properties endow them with the ability to be used in flexible supercapacitors. With a synthesis method such 
as interfacial synthesis, the high mechanical strength and elasticity derived from the tuned microstructures of CPGs can further promote the performance of flexible supercapacitor devices. This concept was demonstrated in the work of Yu et al.,[60] in which PPy hydrogels with structure-derived elasticity were synthesized and used as electrodes in all-solid-state supercapacitors (Figure 8A and $\mathbf{8 B}$ ). The fabricated devices not only showed a high energy storage capacity, with a specific capacitance of $380 \mathrm{~F} \mathrm{~g}^{-1}$, but also demonstrated remarkable flexibility. The capacitance change was negligible even in a highly bent state (Figure 8C). This is because the deformation of the PPy backbone during bending could be largely accommodated by the pore space and the PPy hydrogel could strongly adhere to the current collector.

LIBs show limitations in terms of cycle life and charge/discharge rate but possess high storage capacities and high efficiencies and are relatively lightweight and portable compared with supercapacitors; therefore, these batteries dominate the consumer electronics market. Because of the reversible interactions between lithium ions and polymer chains, conductive polymers have also been directly used as both anodic and cathodic electrode materials in LIBs since the 1980s. A comprehensive review by Haas et al. has covered the electrochemistry and material design of conductive polymers for electrode applications.[62] However, conductive polymers usually show poor stability and low conductivity in reduced states, and these shortcomings seriously inhibit their further applications.

In addition to their application as electrode materials in LIBs, conductive polymers are emerging as promising binder materials. To maximize energy use, every active particle in a battery should be perfectly shaped, sized, and wired to the current collector and to the solid or liquid electrolyte.[63] However, the traditional binder system is a binary hybrid, in which the conductive phase is randomly distributed and bottlenecks or poor contacts may impede effective access to some parts of the battery. Unlike the conventional binder system, conductive polymers could serve dual functions by virtue of their combination of the synergetic features of conventional polymers and organic conductors, thus avoiding the potential aggregation of the conductive additives and the detachment of the polymer layer during cycling. More 
importantly, the chemical structures and related physical/chemical properties of conductive polymers can be tuned by modifying the polymer chains. For example, Liu et al.[64] designed a polymer binder with high electron conductivity and mechanical integrity using a systematic method combining chemical synthesis, quantum calculations, and spectroscopic and mechanical testing tools.

The use of CPGs as binder materials is another promising means of enhancing the performance of LIBs. The highly conductive and continuous 3D CPG framework helps to provide a good electrical connection to the particles and a direct connection to the current collector, thereby enabling outstanding rate capability. The hierarchical pores within such an electrode maximize the interfacial area between the active material and the electrolyte and facilitate the transport of ions. Because of the hydrophilic nature of hydrogels, the polymer can strongly adhere to the surfaces of the active particles and form a uniform coating, thereby preventing the aggregation of the active particles. This conformal coating causes every active particle in the battery to be wired to the current collector and to the solid or liquid electrolyte. Recently, a PANI hydrogel was used as a binder material in Si-based LIBs (Figure 8D and 8E).[65] The PANI gel was polymerized in situ to form a bifunctional conformal coating that bonded to the surfaces of the $\mathrm{Si}$ particles via hydrogen bonding and electrostatic interactions between the positively charged PANI and the negatively charged surface oxide. The capacity of this Si nanoparticle-PANI composite electrode varied from $2,500 \mathrm{mAh} \mathrm{g}^{-1}$ to $1,100 \mathrm{mAh} \mathrm{g}^{-1}$ at charge/discharge rates of 0.3 to $3.0 \mathrm{~A}$ $\mathrm{g}^{-1}$ and retained $\sim 91 \%$ of its capacity after 5,000 cycles at a high current density of 6.0 $\mathrm{Ag}^{-1}$.

The concept of a CPG-based binder system for LIBs has been further extended to a ternary system. Yu et al.[66] designed a 3D nanostructured ternary PPy-Si-CNT electrode that exhibited a remarkable reversible capacity of $\sim 1600 \mathrm{mAh} \mathrm{g}^{-1}$ and a capacity retention $>85 \%$ after 1000 cycles. The incorporation of CNTs greatly enhanced the electron transport/conduction capability of the PPy framework by improving both the physical connections and the electrical contacts between the $\mathrm{Si}$ nanoparticles and the CPG framework. Another ternary system was developed by Bao 
et al.,[67] consisting of a PEDOT:PSS hydrogel, CNTs and active nanoparticles such as $\mathrm{TiO}_{2}$ and $\mathrm{Si}$. The $\mathrm{TiO}_{2}$-based electrodes achieved a capacity of $76 \mathrm{mAh} \mathrm{g}^{-1}$, whereas the Si-nanoparticle-based electrodes reached a high areal capacity of $2.2 \mathrm{mAh} \mathrm{cm}$.
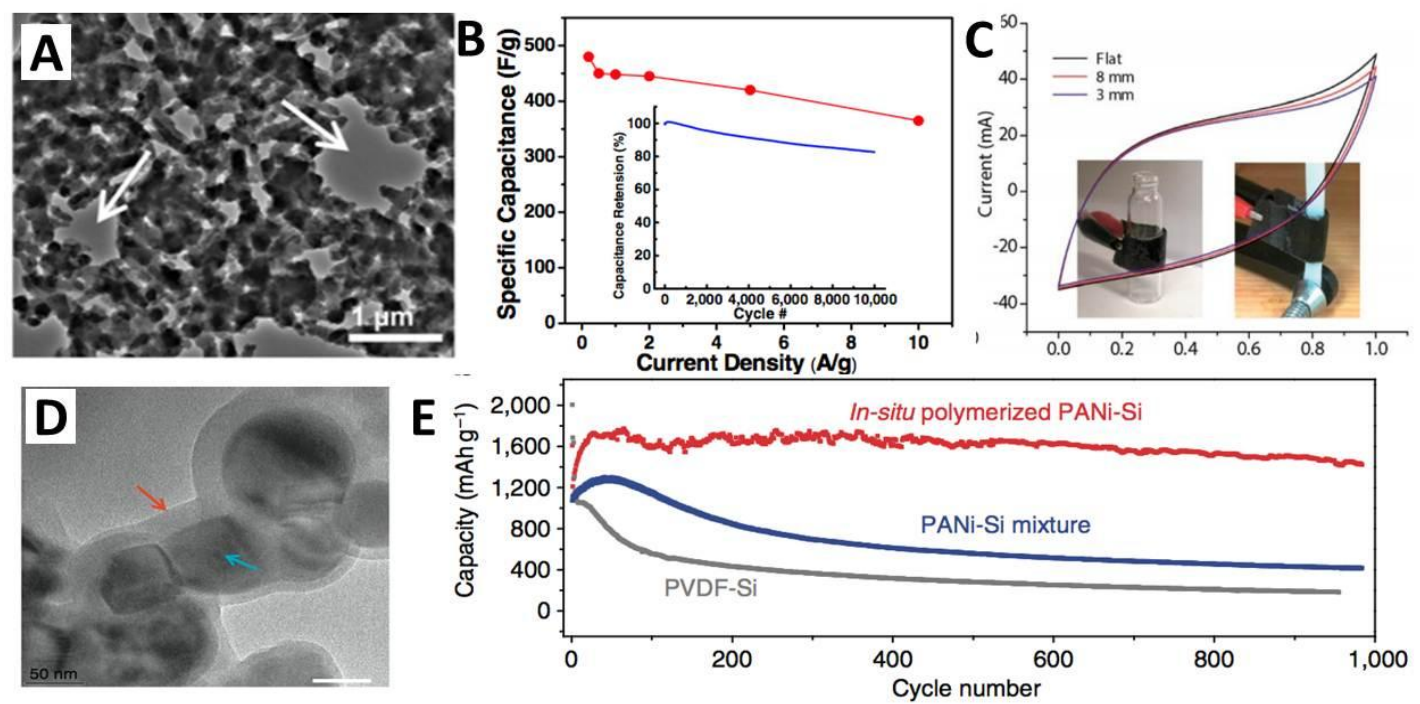

Figure 8. (A) TEM image showing the nanostructured network of a PANI hydrogel. The white arrows indicate micron-size pores in the PANI hydrogel. (B) Summary plot of specific capacitance vs. current density for PANI-hydrogel-based electrodes. (C) CV curves for a PPy-hydrogel-based supercapacitor under different bending conditions. Reproduced with permission from ref. 60. Copyright 2014 Royal Society of Chemistry. (D) TEM image of a composite electrode consisting of porous $\mathrm{Si}$ nanoparticles and a conducting polymer gel. (E) Electrochemical cycling of an in situ polymerized Si particle-PANI composite electrode. Reproduced with permission from ref. 65. Copyright 2013 Nature Publishing Group.

\subsection{CPG-derived carbon frameworks}

CPGs have been investigated as a platform for the development of porous graphitic carbons for energy conversion and storage applications. Compared with traditional porous carbon materials, such as activated carbons, which require extensive purification to eliminate large amounts of impurities and the use of templates to control the pore sizes and pore connections, CPG-derived porous graphitic carbon frameworks can be synthesized via the thermal annealing of CPGs as precursors without any sacrificial template (Figure 9A). CPG-derived porous carbon 
frameworks possess intrinsic hierarchical pore architectures and well-maintained pore connectivity. Elemental doping can be performed concurrently with thermal treatment. Based on these unprecedented properties, CPG-derived carbon frameworks have been exploited for the development of high-performance energy conversion and storage devices.

Bao et al.[68] synthesized porous carbon frameworks via the thermal annealing of phytic-acid-crosslinked PANI hydrogels and used these frameworks to prepare flexible electrodes for micropatterned supercapacitors (Figure 9B and 9C). The relatively small carbon particle size offers scalability and high flexibility in processing. The interconnected fibers provide good mechanical flexibility, resulting in high mass loading. Good electrochemical performance was demonstrated, with high capacitances of 225 and $162 \mathrm{~F} \mathrm{~g}^{-1}$ at current densities of 0.5 and $50 \mathrm{~A} \mathrm{~g}^{-1}$, respectively, and an excellent capacitance retention of $95 \%$ after 10,000 cycles at $5 \mathrm{~A} \mathrm{~g}^{-1}$. The same group also applied porous graphitic carbon for lithium-sulfur batteries.[68] A high specific capacity was achieved and was attributed to the effective hierarchical pore structure and to $\mathrm{N}$ and $\mathrm{O}$ doping, which gave rise to a strong $\mathrm{Li}_{\mathrm{x}} \mathrm{S}$ interaction through which the formation of the lithium sulfide species could be controlled while maintaining high utilization of the active material. In addition to LIBs, a $\mathrm{Zn}$-air battery has been developed by Dai et al.[69] using porous graphitic carbon as an air cathode. The two-electrode primary $\mathrm{Zn}$-air battery showed an open-circuit potential of $\sim 1.48 \mathrm{~V}$, a current density of $\sim 70 \mathrm{~mA} \mathrm{~cm}{ }^{-2}$, and a peak power density of $\sim 55 \mathrm{~mW}$ $\mathrm{cm}^{-2}$, suggesting good catalytic performance of the bifunctional electrocatalyst.

Recently, carbon frameworks derived from CPGs have been applied as catalyst for both the ORR and the OER.[69] CPG-derived carbon frameworks can provide a large number of active sites and can accommodate the linkages of various species-transport channels to their corresponding active sites: voids for $\mathrm{O}_{2}$ and water, polymer chains for protons, and the carbon framework for electrons. The elemental doping can be also tuned by varying the crosslinking molecules and polymer precursors.

Wei et al.[70] synthesized a 3D carbon network from a PANI gel by fixing and 
fully sealing the PANI precursor inside $\mathrm{NaCl}$ via recrystallization. This modified method allows the pore structure to be maintained, resulting in various sizes of pores, from the micro- to the meso- and macro-scales. These pores generate a high density of ORR active sites located along an efficient mass-transport pathway, leading to high conductivity, high ORR activity and good stability in acidic electrolytes. Dai et al. synthesized an N,P-co-doped carbon framework from a phytic-acid-crosslinked PANI hydrogel and demonstrated its bifunctional catalytic activities toward the ORR and OER (Figure 9D and 9E). The minimum overpotentials of the N,P-co-doped carbon framework for the ORR and OER were $0.44 \mathrm{~V}$ and $0.39 \mathrm{~V}$, respectively, lower than those of the theoretical ideal catalysts.

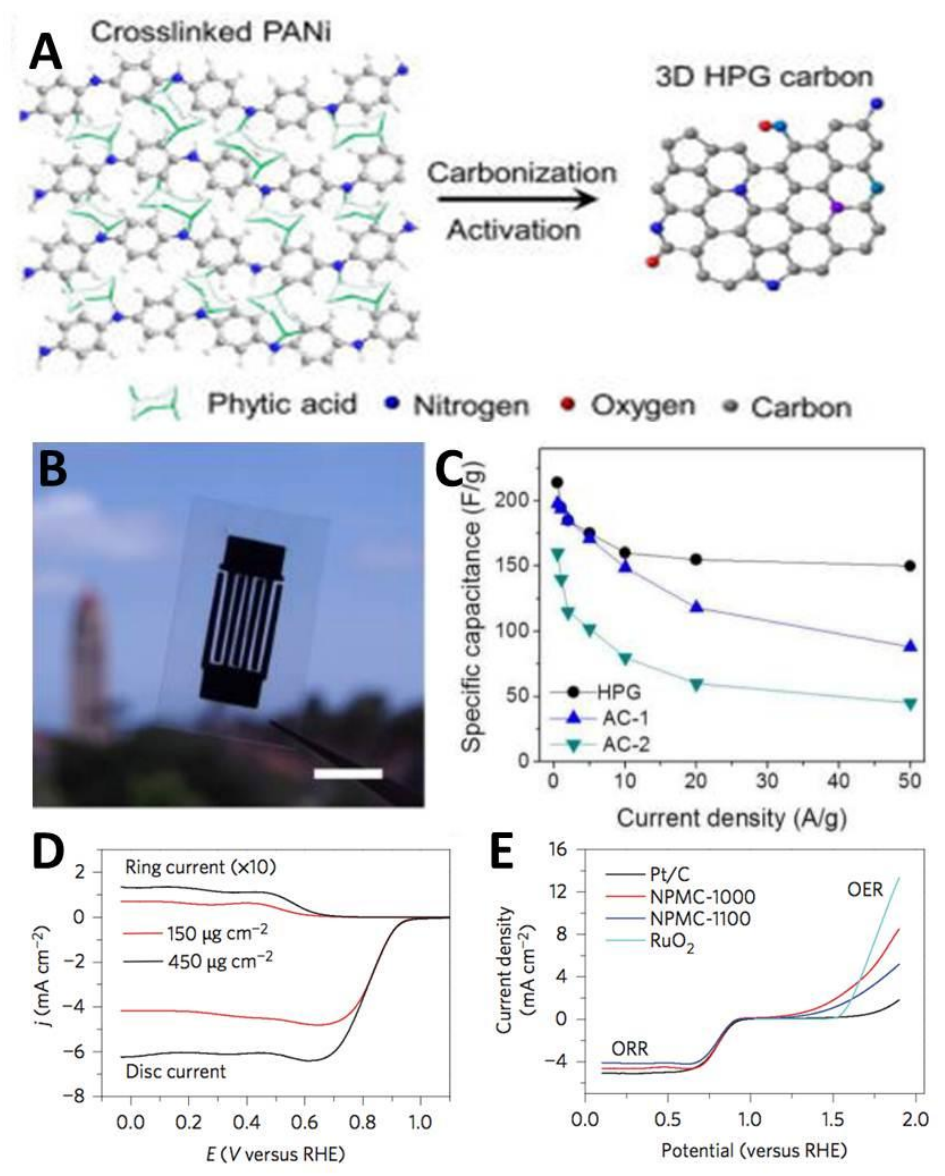

Figure 9. (A) Illustration of the transformation of phytic-acid-crosslinked PANI (left) into doped graphene-like carbon sheets (right). (B and C) Supercapacitor fabricated from a carbon framework on a FET film and the specific capacitance vs. current density curves of supercapacitor electrodes made from different porous carbon frameworks. Reproduced with permission from ref. 68. Copyright 2015 American 
Chemical Society. (D) RRDE measurements of the ORR at N,P-co-doped carbon framework electrodes with different catalyst loadings. (E) An N,P-co-doped carbon framework shows electrocatalytic activities toward both the ORR and the OER. Reproduced with permission from ref. 69. Copyright 2015 Nature Publishing Group.

\subsection{Perspective}

To further improve the energy conversion and storage performance of CPGs and extend their applications, future research should proceed in three directions. First, new strategies must be developed for the fine microstructural control and molecular-level tailoring of CPGs to enhance their mechanical and electrical properties. New crosslinkers and dopants need to be exploited, and novel molecular modification techniques need to be developed to modify the backbones of CPGs, either by adding side chains or through co-polymerization with other polymer sequences. Chemical modifications can be applied to passivate the surfaces of CPGs to avoid undesirable reactions and to improve the electrical and chemical contacts between the polymer matrices and the active materials. Second, hybrid gel materials based on CPGs should be prepared to counteract the disadvantages of CPGs and introduce new features. Hydrogels such as PVA and PEG can help to enhance the mechanical strength and elasticity of such hybrid materials, whereas supramolecular gels and stimuli-responsive polymers can form interpenetrating networks with those of CPGs, thus endowing them with new functions such as stimuli-responsive properties, self-healing properties and enhanced bio-compatibility. These hybrid CPGs could play an increasingly important role in various fields, such as energy storage, biomedical applications, and chemical sensing. Finally, fundamental studies, including simulation and modeling studies as well as studies using state-of-the-art microscopic and spectroscopic techniques, should be conducted to acquire a deeper fundamental understanding of CPGs. Based on such studies, advanced techniques for organic synthesis and novel nanofabrication technologies could be used to achieve delicate control over the electrical, electrochemical, mechanical and surface properties of CPGs. 


\section{Ionically conductive gels for energy applications}

Ionically conductive gels, commonly called gel polymer electrolytes (GPEs), are another class of gels that possess non-conductive polymeric networks but contain movable ions in the liquid phase. Low-molecular-weight solvents are included in 3D networks formed by chemical crosslinking or physical intermolecular forces.[71] Notably, GPEs are different from solid polymer electrolytes (SPEs), in which salts are dissolved not in a solvent but in a polymer matrix, e.g., PEO.[72] GPEs are also fundamentally different from polyelectrolytes, in which the ionic conductivity is provided by charged polymer chains. Based on the plasticizer (liquid phase) that is used, GPEs can be divided into three categories: hydrogel electrolytes (with water), organogel electrolytes (with organic solvents) and ionogels (with ionic liquid).

GPEs have been widely applied in energy storage devices, such as LIBs and supercapacitors. Compared with liquid electrolytes, the quasi-solid-state nature of GPEs avoids the shortcomings of leaking and volatilization. They offer many advantages, such as ease of handling, high reliability and a wide range of operating temperatures. In comparison with glass and ceramic electrolytes, the liquid phase in a GPE enables high ionic conductivity $\left(10^{-4}\right.$ to $10^{-3} \mathrm{~S} \mathrm{~cm}^{-1}$ at room temperature). With their combination of excellent flexibility, liquid-like transport capability and good chemical and electrochemical stability with respect to the electrodes, GPEs are very promising for the development of energy devices with lower costs, improved safety and better packaging processibility.

\subsection{Synthesis of gel polymer electrolytes}

Generally, GPEs can be prepared via three approaches: (1) heating a mixture of a polymer and a plasticizer to form a solution or dispersion, followed by a cooling process in which the viscosity of the mixture increases to form a gel; (2) mixing polymer monomers and plasticizer to form a solution, followed by in situ polymerization initiated by temperature, oxidants, UV light, radiation, etc.; (3) or 
simply swelling a polymer network in a plasticizer solution. In this section, the syntheses of ionically conductive hydrogels, organogels and ionogels are discussed and recent advances are reviewed.

\subsubsection{Hydrogel}

A hydrogel is a kind of 3D structured crosslinked network with abundant hydrophilic groups in its backbone chains or side chains, which can become swollen in water. Because it swells but does not dissolve in water, a hydrogel contains a large amount of water but maintains its shape. Various polymer hosts have been used in hydrogels, including natural products such as agar, chitosan, alginate, gelatin, and carrageenan and synthetic polymers such as PEO, PVA, and polyacrylic acid (PAA). Based on the physical structure of their networks, hydrogels are classified as amorphous, semicrystalline, hydrogen-bonded structures, supramolecular structures or hydrocolloidal aggregates.[73] Based on the nature of the polymer, hydrogels are divided into homopolymeric and copolymeric hydrogels. The hydrophilic homopolymers or copolymers may be soluble in water in their free form, but in a hydrogel, they are rendered insoluble, generally by the presence of covalent, ionic, or physical crosslinks. Hydrogels can be synthesized through either covalent bonding or physical crosslinking, including molecular entanglements, and/or through secondary forces, including ionic bonding, hydrogen bonding or hydrophobic forces.

An ionically conductive hydrogel is limited by its narrow potential window, which is approximately $1.2 \mathrm{~V}$ wide because of the electrochemical splitting of water when used as an electrolyte. However, compared with organic electrolytes, such hydrogels are cheap, safe and environmentally benign. Moreover, because water is the most commonly used solvent, ion conductive hydrogels are highly desirable for use as solid-state electrolytes.

Ionically conductive hydrogels used as electrolytes for energy devices have been reviewed in many articles. In this section, we focus on the recent developments regarding tough hydrogels, which are highly desirable for application in flexible and 
stretchable energy devices. Conventional hydrogels are fragile, which limits their application in flexible and stretchable devices. Gong et al.[74-76] proposed a general route for the formation of strong hydrogels through the construction of a double-network (DN) structure (Figure 10A and 10B). Recently, Suo's group[77] developed a class of stretchable, transparent hydrogels with extreme toughness, which can be stretched beyond 20 times their initial length although they contain $~ 90 \%$ water. These hydrogels were synthesized by mixing two types of crosslinked polymers: ionically linked alginate and covalently crosslinked polyacrylamide (Figure 10C to 10F). Their impressive mechanical properties can be attributed to crack bridging by the network of covalent crosslinks and hysteresis due to the unzipping of the network of ionic crosslinks. These authors also developed polyacrylamide (PAM)-based ionically conductive hydrogels [78, 79] with various chloride salts. Gong et al.[80] proposed a similar mechanism for the production of tough hydrogels via the random copolymerization of oppositely charged ionic monomers around the charge balance point at a high concentration, resulting in a wide distribution of bond strengths. Strong bonds serve as permanent crosslinks, whereas weak bonds can reversibly break and reform, resulting in tough and viscoelastic hydrogels with multiple mechanical properties. 


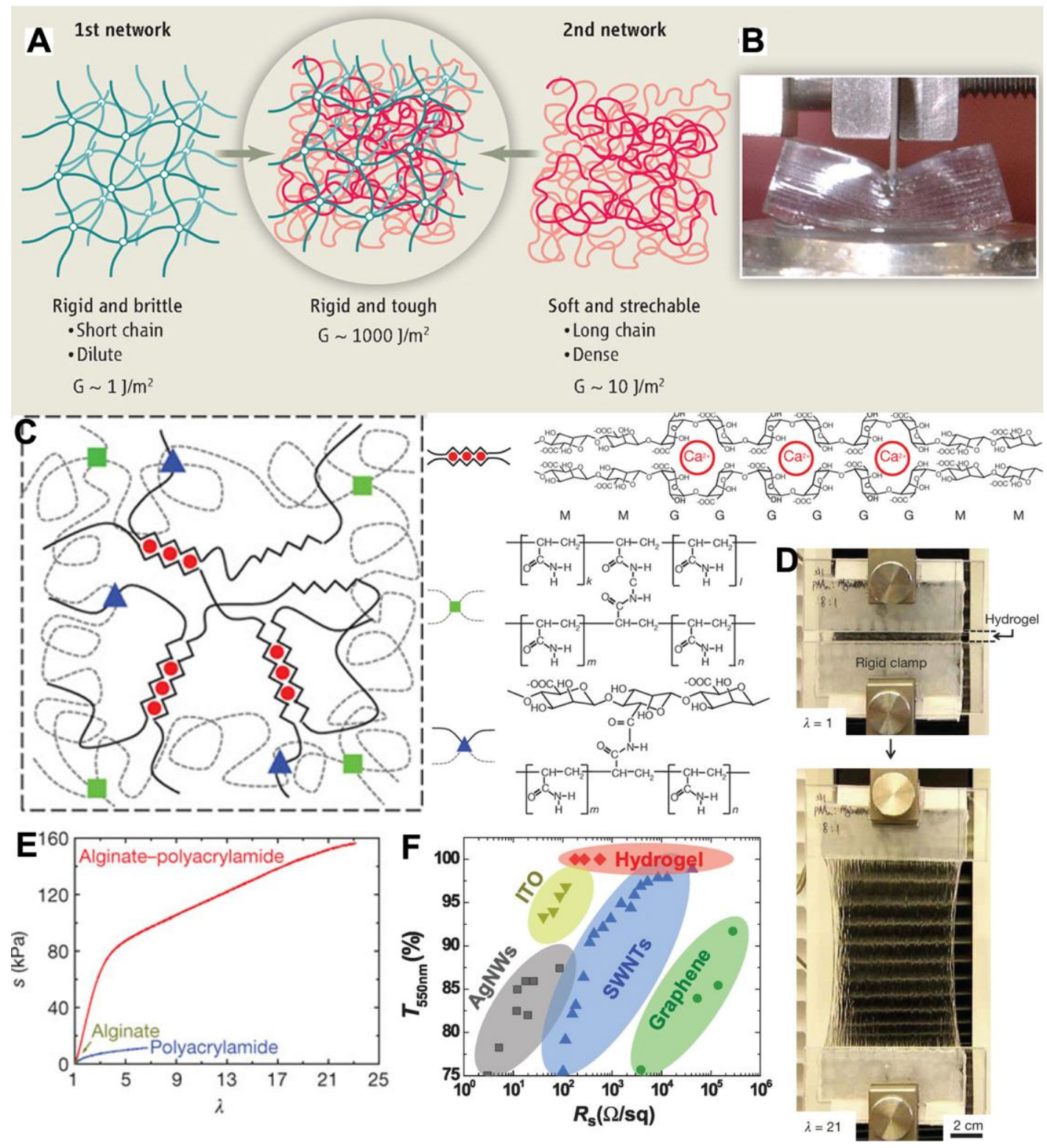

Figure 10. (A) By combining different network materials, tough double-network materials can be created. (B) Photograph of a tough double-network hydrogel containing $90 \mathrm{wt} \%$ of water. The value of the fracture energy $\mathrm{G}$ is an indicator of material toughness. Reproduced with permission from ref. 76. Copyright 2014 American Association for the Advancement of Science. (C) Schematic illustration of an alginate-polyacrylamide hybrid gel; the two types of polymer networks are intertwined and are joined by covalent crosslinks (blue triangles) between amine groups on the polyacrylamide chains and carboxyl groups on the alginate chains. (D) A hydrogel stretched to 21 times its initial length in a tensile machine. (E) Stress-stretch curves of three types of hydrogels. Reproduced with permission from 
ref. 77. Copyright 2012 Nature Publishing Group. (F) Comparison of the transmittance at $550 \mathrm{~nm}$ against the sheet resistance for ionic conductive hydrogels with ITO, AgNWs, SWNTs, and graphene. Reproduced with permission from ref. 78. Copyright 2013 AAAS.

\subsubsection{Organogel}

Organogels have been some of the most extensively investigated solid-state electrolytes for energy storage devices since Feullade and Perche[81] introduced organogel electrolytes into lithium batteries in 1975. Then, in the early 1990s, Bellcore and Sony commercialized a "polymer Li-ion battery" (PLB) using poly(vinylidene fluoride-co-hexafluoropropylene) (PVDF-HFP) as the network.[82] An organogel is a ternary system consisting of a polymer matrix, salt, and low molecular weight polar solvents. Similarly to hydrogel electrolytes, organogel electrolytes are formed by chemical or physical crosslinking. Chemical organogels are covalently crosslinked through thermo/photo-initiated polymerization, which is irreversible, creating permanent crosslinking points. By contrast, physically crosslinked organogels are formed through chain entanglement or regional crystallization, with a microscopic phase separation, which makes no contribution to ionic conductivity.

Organic solvents can reduce the crystallinity of a polymer and therefore lower the glass-transition temperature $\left(T_{\mathrm{g}}\right)$ of the resulting organogel. They should be good solvents with high dielectric constants, low volatilization, and good miscibility with the polymer. Commonly used organic solvents include ethylene carbonate (EC), propylene carbonate (PC), diethyl carbonate (DEC), ethyl methyl carbonate (EMC), dimethyl carbonate (DMC), and dimethyl formamide (DMF). In many cases, a mixture of two or more of these solvents is used to achieve higher ionic conductivity, lower viscosity and a wider potential window. $\mathrm{Li}$ or $\mathrm{Na}$ salts with large anions and low dissociation energies, such as $\mathrm{LiPF}_{6}, \mathrm{LiBF}_{4}, \mathrm{LiCF}_{3} \mathrm{SO}_{3}, \mathrm{LiClO}_{4}, \mathrm{NaClO}_{4}$, and $\mathrm{LiN}\left(\mathrm{CF}_{3} \mathrm{SO}_{2}\right)_{2}$, are commonly used as the supporting electrolytic salts. 
The polymer used as the host matrix should provide a high mechanical stability and good compatibility with the plasticizers. The most commonly used polymers include PAA, PVA, PMMA, poly(vinylidene fluoride) (PVDF), etc. Among them, PVDF has been most extensively explored because it offers the following advantages: (1) good processability for film formation, (2) a high dielectric constant, (3) a high $T_{\mathrm{g}}$ to improve the thermal stability of the organogel, and (4) strong electron-withdrawing C-F groups, which enable high electrochemical stability with respect to the cathode. By adding hexafluoropropylen (HFP) units to form the PVDF-HFP copolymer, a lower degree of crystallinity and a better swelling property in liquid electrolyte can be achieved. Conventionally, PVDF-based solid-state electrolytes are prepared by casting organogels into films without pores, which are not favorable for electrolyte uptake and ionic conductivity. To form porous structures, phase inversion, electrospinning and templating methods are used.[83-85] For example, He et al.[83] proposed a phase inversion process for the preparation of microporous PVDF-HFP membranes using water as the non-solvent. In this process, PVDF-HFP is first dissolved in an acetone/water solution and then cast onto a glass plate to form a film. After evaporation of the solvent, microporous membranes with porosities of up to $70-90 \%$ are directly obtained.

\subsubsection{Ionogel}

An ionogel contains a room-temperature ionic liquid (IL) as the liquid phase. Because the ionic liquid itself has a high ionic conductivity, no additional salts are needed. Ionogels can be divided into two types: physical ionogels, which are cross-linked through weak interactions such as hydrogen bonds, hydrophobic interactions, and crystallite junctions, and chemical ionogels, which are cross-linked through covalent bonding. Physical ionogels are often prepared by incorporating ILs into copolymers, which is a versatile approach because of the ease it offers in tuning the material morphologies and properties by varying block lengths and sequences.[86] In comparison with organogels, ionogels possess several advantages, such as their higher temperature range of operation due to their zero vapor pressure, their inflammability, 
and their environmentally benign nature.

Ionogels are emerging as promising functional gels for use in energy, environmental and catalytic applications. $[87,88]$ Many of their properties can be tuned, including their morphologies, ionic conductivities, moduli, viscoelasticity, and gelatin behavior. Yamamuro et al.[89] found that ionogels consisting of a PMMA network and 1-ethyl-3-methylimidazolium bis(trifluoromethanesulfonyl) (EMITFSI) as the ionic liquid exhibit two $T_{\mathrm{g}}$ values, which drastically decrease as the ionic liquid content increases. Using the quasi-elastic neutron scattering (QENS) technique, these authors established a model in which the plasticizer can be divided into two components: the ionic liquid that is bound to the PMMA and the ionic liquid molecules that are far from the polymer chains. These two components therefore give rise to two glass transition temperatures: a higher and a lower $T_{\mathrm{g}}$. Madsen et al.[90] designed a highly conductive and thermally stable ionogel formed by combining a rigid-rod polyanion, poly(2,2'-disulfonyl-4,4'-benzidine terephthalamide) (PBDT), as the polymer network and an ionic liquid, 1-ethyl-3-methyl-imidazolium trifluoromethanesulfonate $\left(\left(\mathrm{C}_{2} \mathrm{mim}\right)^{+}(\mathrm{TfO})^{-}\right)$, as the plasticizer (Figure 11A to 11D). This ionogel showed a tunable transport anisotropy of up to $3.5 \times$, a high ionic conductivity of up to $8 \mathrm{mS} \mathrm{cm}{ }^{-1}$, high thermal stability up to $300{ }^{\circ} \mathrm{C}$ and a widely tunable modulus from 0.03 to $3 \mathrm{GPa}$. Watanabe et al.[91] synthesized an ionogel based on a self-assembled amphiphilic ABA-triblock copolymer (PSt- $b$-PMMA- $b$-PSt, SMS). An equimolar mixture of tetraglyme (G4) and lithium bis(trifluoromethanesulfonyl)amide (LiTFSA) was used as the plasticizer (Figure 11E and 11F). The solvatophobic A segments contributed to dimensional stability, and the solvatophilic B segments with dissolved [Li(G4)][TFSA] served as tie molecules between the aggregated PSt domains. The self-assembled triblock-based ionogel showed both high Li-ion conductivity and high thermal stability. The same authors also investigated the ion transport properties of ionogels consisting of block copolymers with lamellar morphologies.[92] The confinement- and interface-driven modulation of the ion transport properties of these ionogels was revealed. Dissimilar 
substructures of lamellar microdomains were observed upon varying the type of anion present in the ILs. A wide range of normalized conductivities from 0.03 to 0.6 (theoretical value of 1) were determined by decoupling the segmental motion of the polymer chains from the conductivity. The highest conductivity was achieved when the ILs were confined within ionic domains with sharp interfaces, as a result of the creation of less tortuous ion conduction pathways.

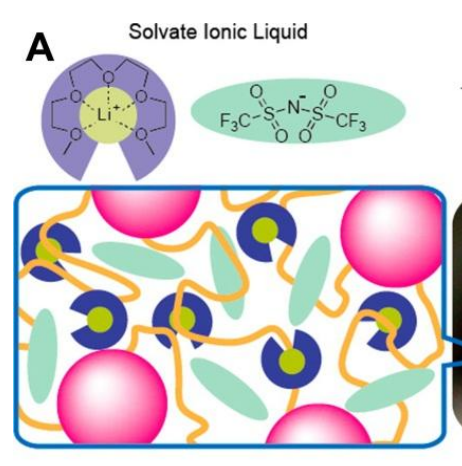

C

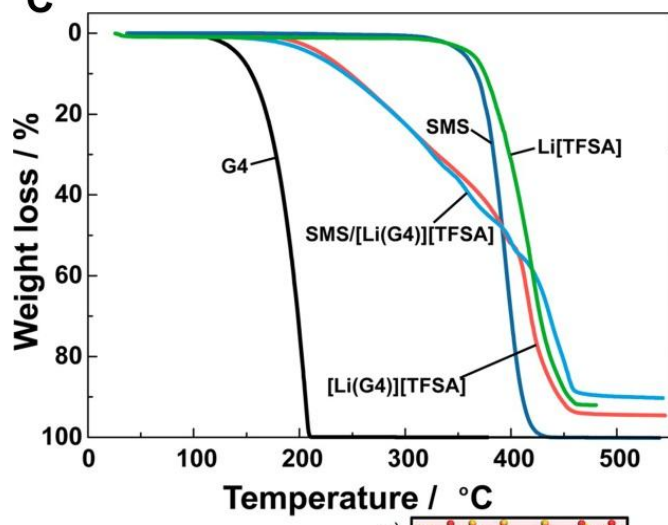

E
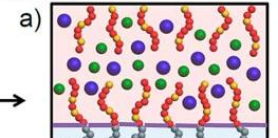

ABA-triblock Copolymer
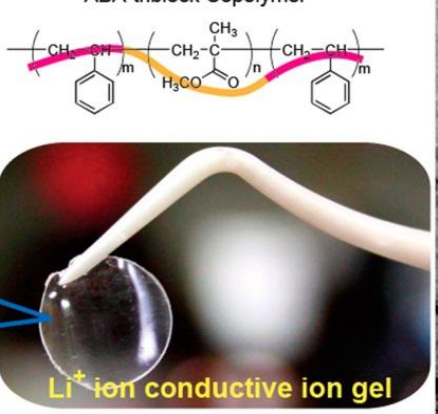

. 60
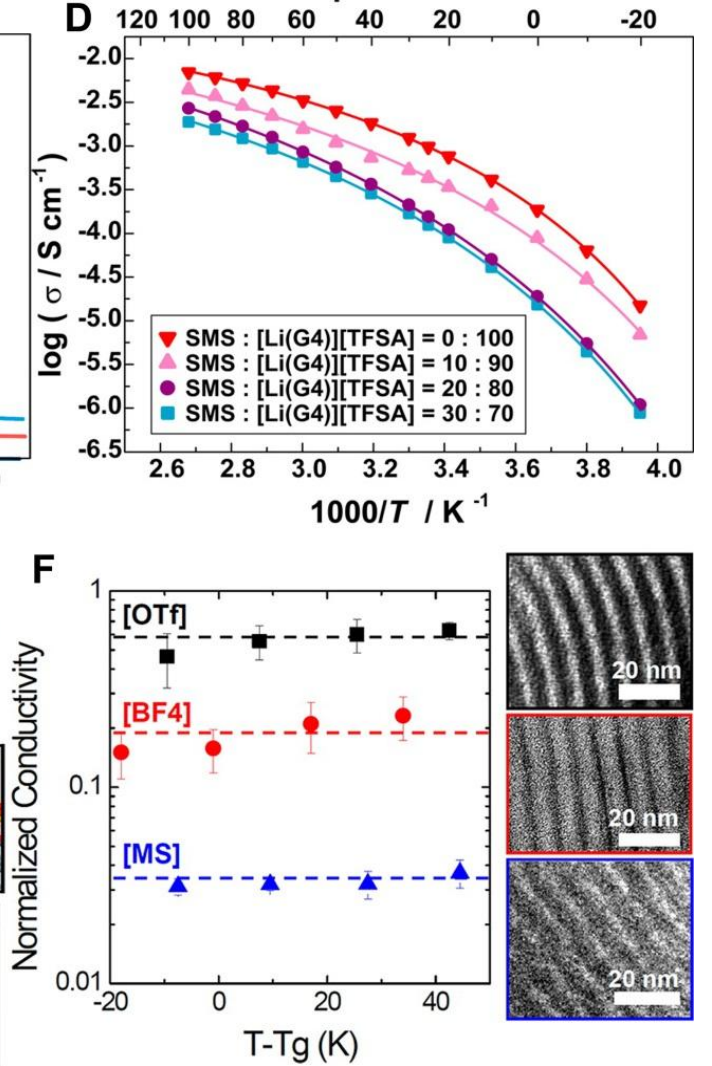

Figure 11. (A) Schematic illustration of an SMS-[Li(G4)][TFSA]-based ionogel and a digital image of the ionogel. (B) TEM image of SMS (1 wt\%) in [Li(G4)][TFSA]. (C) TG curves recorded at a heating rate of $10{ }^{\circ} \mathrm{C} \mathrm{min}^{-1}$ for pure G4, Li[TFSA], the SMS triblock copolymer, [Li(G4)][TFSA] and an ionogel containing $30 \mathrm{wt} \%$ SMS. (D) 
Arrhenius plots of ionic conductivity for SMS-[Li(G4)][TFSA]-based ionogels. Reproduced with permission from ref. 91. Copyright 2014 the American Chemical Society. (E) IL-integrated copolymers with possible substructures of the lamellar microdomains: (a) lamellae with swollen IL-filled PSS phases in which the nonionic PMB domains remain intact; (b) lamellae with laterally expanded PSS phases, resulting in the shrinkage of the PMB layers in the confined spaces; and (c) lamellae with amplified interfacial roughness because of the intermixing of PSS and PMB chains at the interface. (F) The normalized conductivities of IL-incorporated S30MB44(17) copolymers, where the types of anions present in the ILs are noted in the figure. Transmission electron micrographs of IL-containing S30MB44(17) copolymers revealing the dissimilar internal structures of the lamellar morphologies are shown in the insets, in which the PSS phases have been darkened by means of $\mathrm{RuO}_{4}$ staining. Reproduced with permission from ref. 92. Copyright 2013 the American Chemical Society.

\subsection{GPEs for energy storage and conversion}

As discussed previously, GPEs possess many advantages in comparison with liquid electrolytes. Therefore, GPEs have been widely used in fuel cells, solar cells, secondary batteries and electrochemical capacitors and exhibit superior performance in flexible solid-state electrochemical devices.

\subsubsection{Supercapacitors}

GPEs, especially hydrogel electrolytes, have been extensively explored for use in supercapacitors. Detailed descriptions of the progress in this area can be found in several recent review articles.[71, 93, 94] Among the GPEs utilized in supercapacitors, PVA-based hydrogel electrolytes, such as $\mathrm{PVS}_{2} \mathrm{H}_{2} \mathrm{SO}_{4}, \mathrm{PVA}-\mathrm{H}_{3} \mathrm{PO}_{4}, \mathrm{LiCl}$ and PVA-KOH, have attracted considerable attention because they can be used as frameworks in a wide $\mathrm{pH}$ range. The electrochemical behavior of pseudocapacitive electrode materials is strongly dependent on the nature of the electrolyte. The interfacial matching between electrodes and PVA-based hydrogel electrolytes has 
been optimized. Rajagopalan et al.[95] used PVA- $\mathrm{H}_{2} \mathrm{SO}_{4}$ hydrogel membranes with an ionic conductivity of $\sim 45 \mathrm{mS} \mathrm{cm}$ cm $^{-1}$ as electrolytes and single-walled carbon nanotubes as electrodes for the fabrication of flexible EDLCs, which showed high power densities of up to $1040 \mathrm{~kW} \mathrm{~kg}^{-1}$ and excellent cycle stability. Zhang et al.[96] reported PVA-potassium borate hydrogel membranes formed on active carbon electrodes via electrodeposition. These hydrogel membranes served as both the electrolyte and the separator in supercapacitors, showing lower internal resistance and better electrochemical performance compared with a liquid electrolyte. Wang et al.[97] used PVP as the pore-forming agent to enhance the swelling ratio of PVA in an $\mathrm{H}_{2} \mathrm{SO}_{4}$ electrolyte and glutaraldehyde (GA) as the cross-linking agent to solve the fluidity and thermal aging problems of PVA at high temperatures. Wu et al.[98] synthesized a redox-mediated GPE by adding p-benzenediol into a conventional $\mathrm{PVA}-\mathrm{H}_{2} \mathrm{SO}_{4}$ hydrogel. The redox reaction of p-benzenediol/p-benzoquinone and its quick electron relay at the electrolyte/electrode interface efficiently improved the electrochemical performance of a supercapacitor with activated carbon electrodes. In addition to PVA-based hydrogels, other hydrogels such as $\mathrm{PAA} / \mathrm{H}_{2} \mathrm{SO}_{4}$ and $\mathrm{PAAK} / \mathrm{H}_{2} \mathrm{SO}_{4}$ as well as organogels and ionogels based on PEO, PMMA, PVP, and PVDF have also been used in supercapacitors. For example, crosslinked PVDF-HFP-based porous organogels have been employed in supercapacitors.[99, 100] A PMMA-based organogel can protect $\delta-\mathrm{MnO}_{2}$ nanowires from fracture, delamination, and separation from conductive substrates, resulting in extraordinary cycle stability for up to 200,000 cycles.[101] An ionogel based on a poly(styrene-block-ethylene oxide-block-styrene) (PS-PEO-PS) triblock copolymer and an ionic liquid of 1-ethyl-3-methylimidazolium bis(trifluoromethylsulfonyl)imide [EMIM] $\left[\mathrm{NTf}_{2}\right]$ has also been used as both separator and electrolyte for all-solid-state flexible supercapacitors.[102]

Because of the growing interest in portable and wearable electronic devices, there is an urgent need to develop flexible, stretchable and embeddable energy storage devices, in which GPEs can play a very important role. Ha et al.[103] fabricated a stretchable and patchable array of micro-supercapacitors (MSCs) using a 
PMMA-based GPE with a stable potential of between 0 and $1.2 \mathrm{~V}$. These MSCs maintained over $85 \%$ of their initial capacitance for two weeks in ambient air without encapsulation. The MSCs could be further dry-transferred onto deformable Ecoflex substrates to form $2 \times 4$ arrays, which demonstrated stable electrochemical performance under bending, twisting, and stretching deformation. The incorporation of nanoparticles into GPEs may result in enhanced mechanical strength, thereby making them more favorable for use in stretchable devices. Loh et al.[104] presented three designs for combining nanoparticles with hydrogels: (1) the stabilization of inorganic or polymer nanoparticles on micro- or nanoscale hydrogels, (2) the immobilization of nanoparticles on a hydrogel matrix via noncovalent interactions and (3) similar immobilization via covalent interactions. Recently, Zhi et al.[105] synthesized a stretchable and self-healable hydrogel electrolyte comprising PAA crosslinked via hydrogen bonding and vinyl hybrid silica nanoparticles (VSNPs-PAA) (Figure 12A to 12D). Ionic conductivity of up to $\sim 7 \mathrm{mS} \mathrm{cm}^{-1}$ was achieved for this hydrogel using an aqueous $\mathrm{H}_{3} \mathrm{PO}_{4}$ solution as the plasticizer. The hydrogel could be stretched greater than $3,700 \%$ without cracking. It could be easily self-repaired at room temperature without any decrease in ionic conductivity. Supercapacitors assembled using this hydrogel and PPy-deposited carbon nanotube (CNT) paper electrodes exhibited $\sim 100 \%$ efficiency of self-healability during 20 breaking/healing cycles and withstood $600 \%$ strain with enhanced performance. Wang et al.[106] synthesized tough gels consisting of four components: 1-ethyl-3-methyl-imidazolium chloride (EMIMCl), poly(hydroxyethyl methacrylate) (PHEMA), chitosan and water (Figure 12E to 12I). The tough physical gelation of the EMIMCl gel was ascribed to hydrogen bonding between the hydroxyl groups in the hydroxyethyl methacrylate (HEMA), the amine groups of the chitosan and the imidazolium groups in the solvent. This gel can accommodate between 5 and $50 \mathrm{wt} \%$ water with a slight decrease in strength but a marked increase in ionic conductivity. 


\section{A}
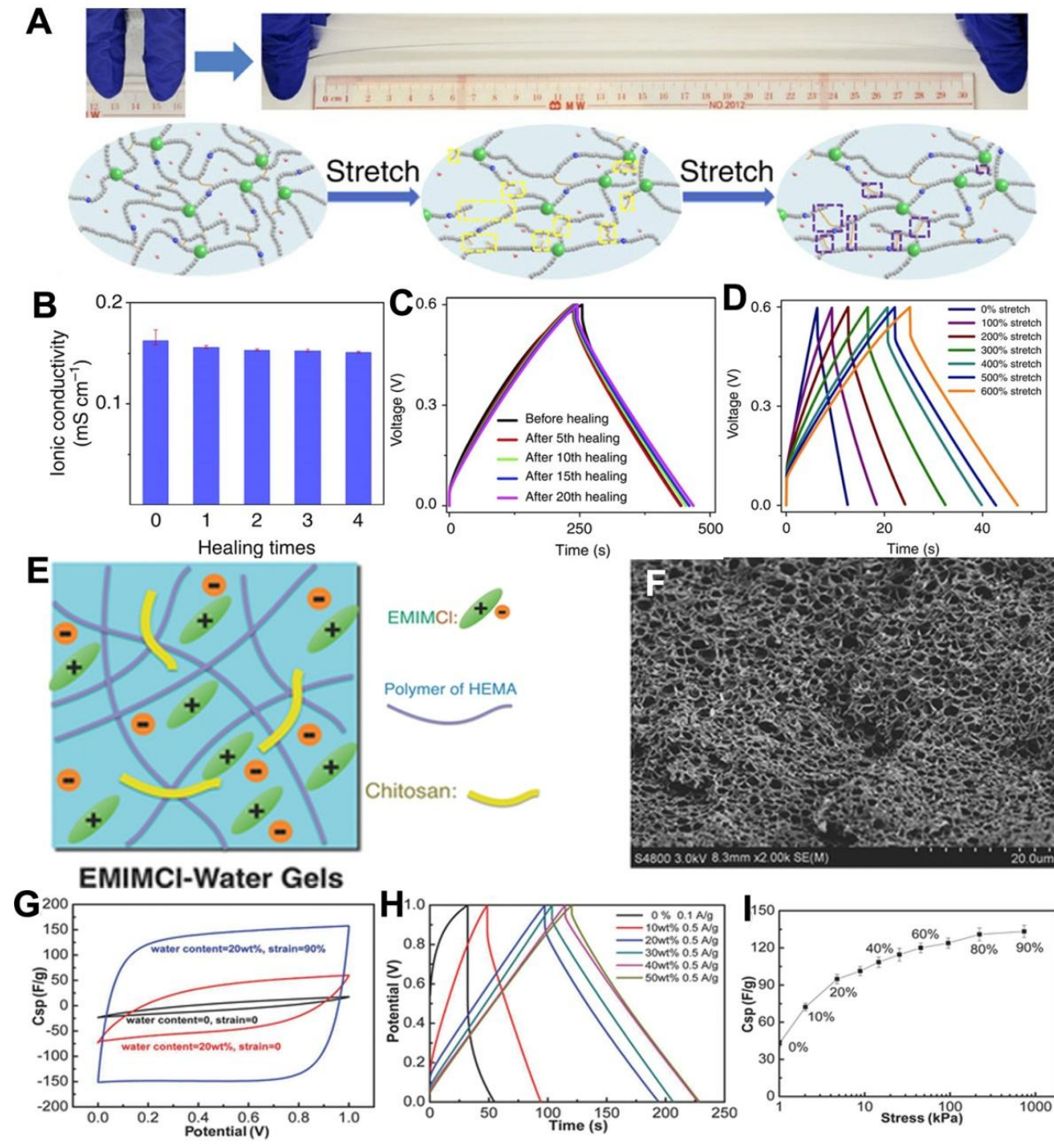

Time (s)
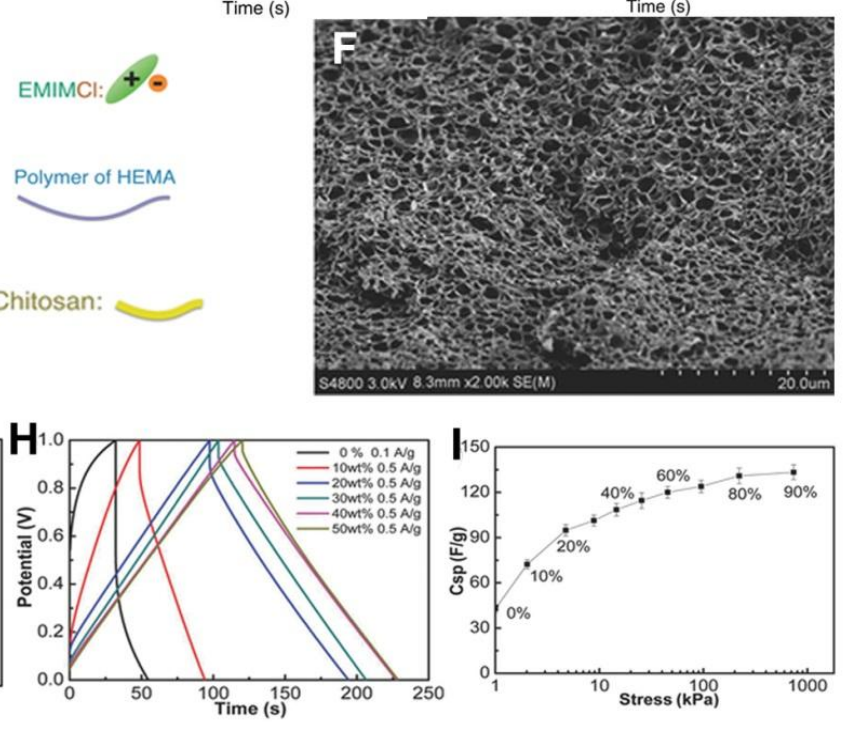

Figure 12. (A) Relaxed and elongated states of VSNPs-PAA and a schematic illustration of the origin of its super-stretchablity. (B) Ionic conductivity after multiple breaking/healing cycles. (C) Self-healing performance of a supercapacitor at a charging/discharging current of $1 \mathrm{~mA}$. (D) Galvanostatic charge-discharge (GCD) curves from 0 to $600 \%$ strain. Reproduced with permission from ref. 105. Copyright 2015 Nature Publishing Group. (E) The schematic structure of a PHEMA/EMIMCl hydrogel. (F) SEM image of the PHEMA/EMIMCl gel after supercritical drying. (G) Cyclic voltammetry curves from 0 to $90 \%$ compression of the PHEMA/EMIMCl hydrogel with $20 \mathrm{wt} \%$ water. (H) GCD curves for the PHEMA/EMIMCl hydrogel with 0 to $50 \mathrm{wt} \%$ water under $90 \%$ compression. (I) Specific capacitances of a PHEMA/EMIMCl-hydrogel-based supercapacitor with $20 \mathrm{wt} \%$ water under various compressive stresses. Reproduced with permission from ref. 106. Copyright 2014 
WILEY-VCH Verlag Gmbh \& Co. kGaA, Weinheim.

\subsubsection{Lithium batteries}

Over the past two decades, LIBs have been widely used as energy sources in consumer electronics such as cell phones and laptop computers. They are also considered to be the most promising power sources for electric vehicles and storage devices for intermittent energy sources such as wind and solar energy. However, the use of organic liquid electrolytes in these batteries could lead to severe safety concerns, including risks of leakage, fire and even explosion. Organogel-based electrolytes are promising substitutes for organic liquid electrolytes because they possess comparable ionic conductivities, wide potential windows, good compatibility with electrodes and thermal stability. Several review articles have discussed organogels for LIBs in detail.[72, 107, 108]

Considerable advances in mechanical strength, ionic conductivity, and electrochemical and thermal stability have been achieved since the first introduction of organogels into LIBs. The strategies for improvement include the addition of nanoparticles, copolymerization, chemical crosslinking and blending. It has been found that the incorporation of inorganic nanoparticles fillers with high surface areas, such as $\mathrm{SiO}_{2}, \mathrm{TiO}_{2}$, and $\mathrm{Al}_{2} \mathrm{O}_{3},[109,110]$ into GPEs can enhance their strength, ionic conductivity and interfacial compatibility with electrodes. For example, Kim et al.[111, 112] incorporated methacrylate- or vinyl-functionalized $\mathrm{SiO}_{2}$ nanoparticles into PAN membranes. The methacrylate groups on the $\mathrm{SiO}_{2}$ nanoparticles reacted with the tri(ethylene glycol) diacrylate (TEGDA) gelator, forming a crosslinked composite GPE with high ionic conductivity and favorable interfacial characteristics. $\mathrm{LiNi}_{0.8} \mathrm{Co}_{0.15} \mathrm{Al}_{0.05} \mathrm{O}_{2}$-graphite cells using crosslinked PAN-SiO${ }_{2}$ GPEs with enhanced thermal stability exhibit high discharge capacity and good capacity retention at both ambient temperatures and elevated temperatures (Figure 13A to 13D). By replacing the nonporous $\mathrm{SiO}_{2}$ nanoparticles in the GPE with mesoporous $\mathrm{SiO}_{2}$ nanoparticles functionalized with methacrylate (MA), the resulting $\mathrm{LiNi}_{1 / 3} \mathrm{Co}_{1 / 3} \mathrm{Mn}_{1 / 3} \mathrm{O}_{2}$-graphite cells can be made to exhibit a much improved rate capability because mesoporous 
MA-SiO 2 -based GPEs are more effective for Li-ion transport and for maintaining good interfacial contacts with the electrodes (Figure 13E to 13G).

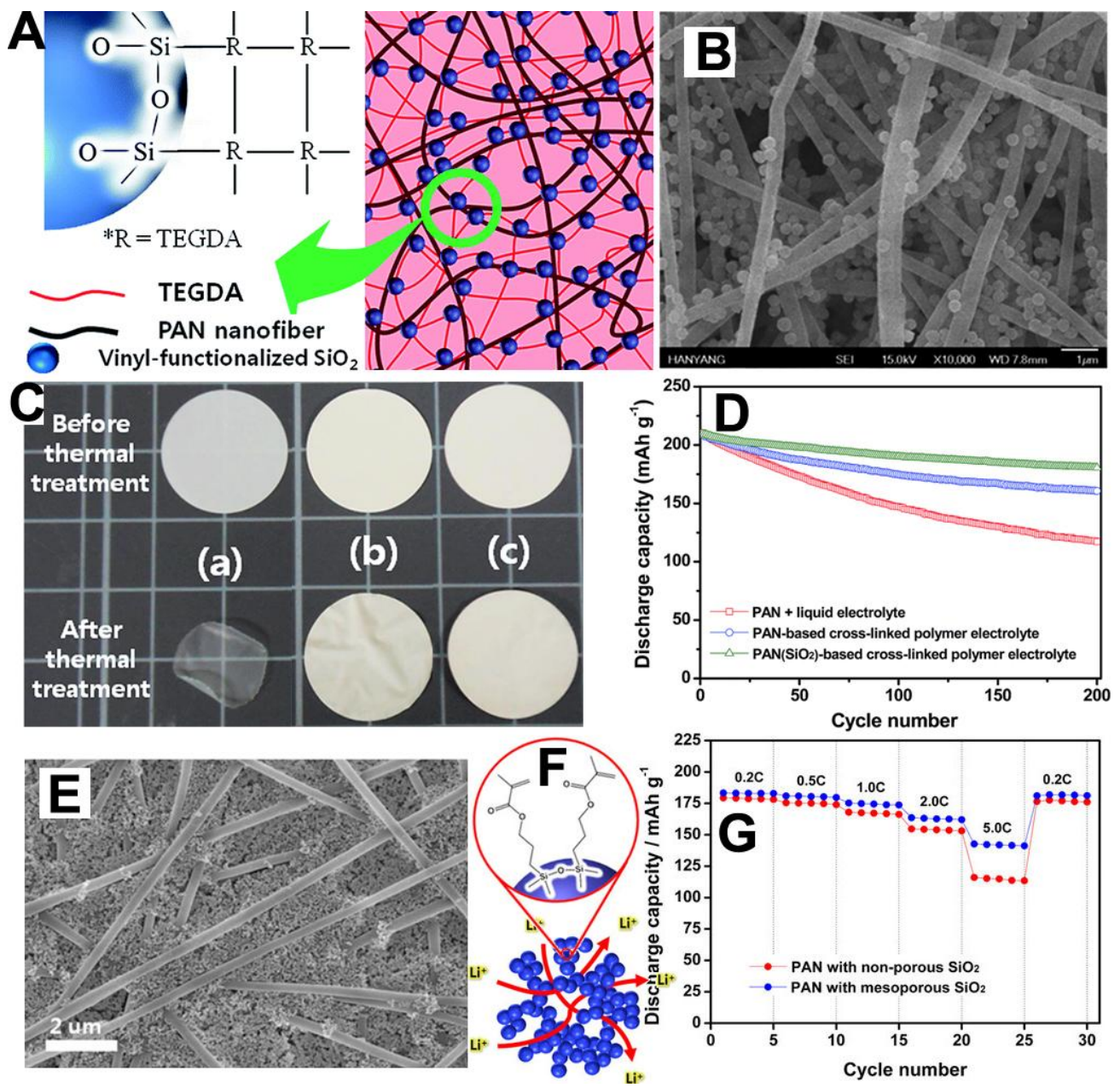

Figure 13. (A) Schematic illustration of a crosslinked GPE with a fibrous PAN membrane, vinyl-functionalized $\mathrm{SiO}_{2}$ nanoparticles and TEGDA. (B) SEM image of a fibrous PAN membrane with vinyl-functionalized $\mathrm{SiO}_{2}$ nanoparticles. (C) Photographs of (a) a polyethylene separator, (b) a fibrous PAN membrane, and (c) a fibrous PAN membrane with vinyl-functionalized $\mathrm{SiO}_{2}$ nanoparticles before and after exposure at $150{ }^{\circ} \mathrm{C}$ for $1 \mathrm{~h}$. (D) Discharge capacities at $0.5 \mathrm{C}$ of LIBs assembled with different GPEs at $55^{\circ} \mathrm{C}$. Reproduced with permission from ref. 104. Copyright 2016 Nature Publishing Group. (E) SEM image of a PAN membrane with mesoporous MA-SiO 2 nanoparticles. (F) Schematic illustration of lithium-ion transport behavior after the introduction of mesoporous $\mathrm{MA}-\mathrm{SiO}_{2}$ nanoparticles into a crosslinked GPE. (G) Discharge capacities of LIBs assembled with different GPEs as a function of the 
C rate. Reproduced with permission from ref. 112. Copyright 2015 Royal Society of Chemistry.

Chemical crosslinking is also a powerful method of improving the mechanical properties of GPEs. For example, PVDF-HFP has been crosslinked with polyethylene glycol dimethacrylate (PEGDMA) or trimethylolpropane triacrylate (TMPTA) to enhance its thermal and dimensional stability through thermal curing or ${ }^{60} \mathrm{Co}$ radiation. $[99,113]$ The mechanical strength and/or ionic conductivity of a GPE can be further adjusted by blending an additional polymer into the main network. Yang et al.[114] reported a semi-interpenetrating polymer network (Semi-IPN) organogel based on a highly crosslinked poly(ethylene glycol) diacrylate-co-poly(vinylene carbonate) (PEGDA-co-PVC) network with a linear PVDF-HFP polymer produced via UV light curing. This Semi-IPN-based organogel showed high toughness and flexibility, with superior thermal stability and excellent interfacial stability with respect to metal electrodes. Moreover, copolymerization can allow the intrinsic properties of a polymeric network to be adjusted through the introduction of monomers with different characteristics. Kao et al.[115] designed a highly conductive organogel based on poly(propylene glycol)-block-poly(ethylene glycol)-block-poly(propylene glycol) bis(2-aminopropyl ether) (ED2003), poly(ethylene glycol) diglycidyl ether (PEGDGE), 2,4,6-trichloro-1,3,5-triazine (cyanuric chloride, CC), PVDF-HFP and 3-(glycidyloxypropyl)trimethoxysilane (GLYMO). This hybrid organogel showed a remarkable swelling ratio of up to $800 \%$ and a high ionic conductivity of $6.9 \mathrm{mS} \mathrm{cm}{ }^{-1}$ with $\mathrm{LiClO}_{4}$ in $\mathrm{EC} / \mathrm{PC}$.

The properties of the interfaces between the electrolytes and electrodes in lithium batteries are crucial for their performance, especially in newer types of lithium batteries, such as redox-active organic-material-based batteries, Li-S batteries and Li- $\mathrm{O}_{2}$ batteries. GPEs are promising for use in these applications because of their unique quasi-solid-state nature and ease of structural tailoring. For example, an organogel based on PMA/PEG-LiClO $4 / \mathrm{DMSO}$ (where PMA denotes poly(methacrylate)) was used as the electrolyte for LIBs with organic carbonyl 
compound cathodes (calix[4]quinine, C4Q) exhibiting multi-electron reactions.[116] Hammond et al.[117] examined the stability of PAN, PVC, PVDF, PVDF-HFP and PVP in the presence of $\mathrm{Li}_{2} \mathrm{O}_{2}$, which is a main product of a $\mathrm{Li}-\mathrm{O}_{2}$ battery. Lee et al.[118] reported a PVDF-HFP-based organogel with $50 \mathrm{wt} \% \mathrm{Al}_{2} \mathrm{O}_{3}$ powder for use as the composite protective layer (CPL) for a Li metal foil. Using an integration strategy that combined a redox-mediator-containing electrolyte with a CPL-protected Li anode, these authors demonstrated $\mathrm{Li}-\mathrm{O}_{2}$ batteries with enhanced cycle stability and improved efficiency during cycling. It is also worth noting that lithium batteries, including $\mathrm{Li}-\mathrm{S}$ and $\mathrm{Li}_{2} \mathrm{O}_{2}$ batteries, in which metallic lithium is used as the anode suffer from severe safety concerns because of the growth of lithium dendrites.[119] This problem is expected to be solved with the use of solid-state electrolytes. Given that the ionic conductivity of typical solid-state electrolytes cannot meet the necessary requirements, GPEs with enhanced mechanical strength could be suitable candidates. For example, it has been reported that by incorporating $\mathrm{SiO}_{2}$ particles into poly(lithium acrylate), lithium dendrite growth can be greatly suppressed.[120]

Ionogels have recently been emerging as a new type of GPEs for use in lithium batteries. Zhou et al.[121] cross-linked the ionic liquid 1-ethyl-3-methylimidazolium bis(trifluoromethylsulfonyl) $\left(\left[\mathrm{C}_{2} \mathrm{C}_{1} \mathrm{im}\right]\left[\mathrm{NTf}_{2}\right]\right)$ with a single-walled carbon nanotube network to form an ionogel (CNG). This ionogel, as an air electrode, allows efficient 3D tricontinuous passage of electrons, lithium ions and oxygen, showing high reversibility for use in $\mathrm{Li}-\mathrm{O}_{2}$ and $\mathrm{Li}$-air batteries. PVDF-HFP blended with PMMA has been used as the network for an ionogel with 1-butyl-3-methylimidazolium tetrafluoroborate $\left(\mathrm{BMIMBF}_{4}\right)$ as the plasticizer.[122] The morphology, porosity, $T_{\mathrm{g}}$ and ionic conductivity of the resulting ionogel can be tuned by adjusting the percentage of PMMA. Polyvinyl acetate (PVAc) can be also used to improve the thermal stability and electrochemical window of PVDF-BMIMBF 4 ionogels.[123]

\subsubsection{Solar cells}

Methods of harvesting solar energy with high efficiency are currently a very active 
area of research. Dye-sensitized solar cells (DSSCs) with power conversion efficiencies $(\eta)$ of up to $12.3 \%$ have been produced using $\mathrm{I}^{-} / \mathrm{I}^{3-}$ and $\left[\mathrm{Co}(\mathrm{bpy})_{3}\right]^{2+/ 3+}$ redox mediators.[124] However, the practical long-term use of DSSCs is hindered by the use of volatile and leak-prone organic solvents. GPEs have attracted considerable attention because of their excellent liquid uptake ability and long-term stability. Organogels based on various polymers for use in DSSCs have been reported, such as PVDF-HFP,[125] poly(acrylonitrile-co-vinyl acetate) (PAN-VA),[126] and bisphenol A ethoxylate dimethacrylate-poly(ethylene glycol) methyl ether methacrylate (BEMA-PEGMA).[127]

Limited by their ionic conductivity, the efficiencies of DSSCs that incorporate GPEs are still lower than those with liquid electrolytes ( $8 \%$ vs. 12\%), and further efforts in this direction are needed. Ho et al.[128] designed a GPE based on a poly(oxyethylene)-segmented amide-imide (POE-PAI) network for DSSCs with $\mathrm{TiO}_{2}$ electrodes. Electrochemical impedance spectra showed that with an optimized polymer ratio, lower resistances than that of a liquid electrolyte could be obtained for this GPE. Thus, a $\eta$ of $9.48 \%$ was achieved, which was higher than the corresponding value for a liquid electrolyte (8.84\%). Recently, Khanmirzaei et al.[129] used a nonvolatile IL of 1-methyl-3-propylimidazolium iodide (MPII) as a plasticizer. Upon the combination of this IL with hydroxypropyl cellulose (HPC), sodium iodide, ethylene carbonate (EC) and propylene carbonate (PC), the as-formed nonvolatile GPS system showed a high ionic conductivity of up to $7.37 \mathrm{mS} \mathrm{cm}^{-1}$, and a $\eta$ of $5.79 \%$ was achieved.

Another obstacle facing GPEs is their lower ability to penetrate into the porous structure of an electrode. To overcome this disadvantage, post-polymerization after penetration of the monomers into the electrodes has been proposed. However, this approach requires high temperatures or additives to induce polymerization, which may be harmful to the DSSCs. Nevertheless, Park et al.[130] reported a method of post-polymerization for GPEs based on the controlled dissolution of polystyrene nanobeads on the counter electrode, resulting in a good penetration ability. The 
optimal DSSC fabricated with such a GPE exhibits a nearly identical $\eta$ to that of a liquid-electrolyte-based DSSC but a greatly improved long-term stability.

\subsubsection{Fuel cells}

GPEs are used as polymer electrolyte membranes (PEMs) in fuel cells, namely, in PEMFCs. Whereas conventional PEMs suffer from ready dehydration, crosslinked hydrogels have found to be good candidates for PEMs. Sahai et al.[131] synthesized a PEM consisting of an ionically crosslinked chitosan hydrogel for direct borohydride fuel cells (DBFCs) with high performance and eco-friendliness. A polyethylenimine-based hydrogel with viologen modification has been used as a protective support for hydrogenase against high-potential deactivation and oxygen damage in biofuel cells.[132] Sahai et al.[133] also synthesized ammonium-functionalized silica-PVA crosslinked PEMs as anion-exchange membranes (AEMs) for application in direct methanol fuel cells (DMFCs). Because of their quaternary ammonium group functionality, these AEMs exhibit low methanol permeability along with a hydroxyl ion conductivity of $75.7 \mathrm{mS} \mathrm{cm}^{-1}$.

Ionogels with good thermal stability, high ionic conductivity and gas permeability have become promising candidates for use as separators in fuel cells. For example, ionogels based on sulfonated polyimide and diethylmethylammonium trifluoromethanesulfonate as a proton conductor have been developed for use as electrolytes in $\mathrm{H}_{2} / \mathrm{O}_{2}$ fuel cells that can be operated at temperatures higher than $100{ }^{\circ} \mathrm{C}$ under non-humidified conditions.[134]

\subsection{Perspective}

Ionically conductive gels are widely used in electrochemical energy storage/conversion devices. Although great progress has been made in the field of GPEs, this field is still facing considerable challenges because of the growing demand for flexible devices with high energy densities. The general requirements for GPEs intended for use in energy storage/conversion devices include (1) high ionic conductivity, (2) good mechanical strength, (3) good electrochemical and thermal 
stability in the potential windows of operation for specific electrodes, and (4) high safety and reliability for large-scale utilization. To improve these properties, various techniques for the structural and compositional design of GPEs have been developed, including filling with nanoparticles, chemical crosslinking, copolymerization, and polymer blending. However, improvements in mechanical strength sometimes come at the cost of ionic conductivity. It will be important to gain a further understanding of the fundamental relationships among polymer networks, plasticizers and salt ions through theoretical and experimental investigations.

Meanwhile, the development of novel gel systems will open up new possibilities regarding ionic conductive gels for energy applications. For example, supramolecular gels, which rely on non-covalent interactions (such as hydrogen bonds or electrostatic interactions) to assemble smaller building blocks into rigid networks, demonstrate the advantages of facile, rapid and reproducible preparation and self-healing capabilities. Furthermore, because of the highly tunable and multifunctional characteristics of gels, design frameworks for the incorporation of active species such as inorganic nanoparticles, molecular species, and nanocatalysts will give rise to new and exciting properties for potential applications in energy storage and conversion.

\section{Inorganic gels for energy applications}

\subsection{Synthesis of inorganic gels}

The 3D structures of inorganic materials have been studied intensively, not only because of their interesting intrinsic properties, which arise from their low dimensionality and the quantum effects of the building blocks at the nano-level, but also because of their capability for direct integration into nanoscale systems. Among the various 3D structured inorganic materials, porous inorganic hydrogels/aerogels are highly attractive for use in applications related to energy conversion and storage, sensing, catalytic conversion, selective absorption and removal, or thermal insulation, among others, by virtue of their large pore volumes, high specific surface areas, low 
bulk densities, and low thermal conductivities. Inorganic hydrogels/aerogels can be directly used as self-supported active electrodes or catalysts in energy conversion and storage devices. Their large surface areas ensure good contact between the active materials and electrolyte and provide a large number of active sites for electrochemical reactions. Their porous structures also facilitate the flow of liquid electrolytes, thus enhancing the reaction kinetics.

Several synthesis strategies have been developed for the preparation of inorganic gel materials, and many inorganic precursors have been used. Sol-gel routes have been widely used to synthesize transition and main-group metal oxide aerogels. In the work of Gash et al.,[135] epoxides were adopted as gelation agents for the sol-gel synthesis of aerogels from simple metal ion salts, including $\mathrm{Fe}^{3+}, \mathrm{Al}^{3+}, \mathrm{In}^{3+}, \mathrm{Ga}^{3+}, \mathrm{Zr}^{4+}$, $\mathrm{Hf}^{4+}, \mathrm{Ta}^{5+}, \mathrm{Nb}^{5+}$, and $\mathrm{W}^{6+}$. The gel formation and its rate depended on the solvent used, the precursor salt, the epoxide/metal-ion ratio, and the type of epoxide employed. The driving forces of gelation were considered to be the coordination between metal ions and strong electrostatic interactions.

Template-guided methods have been developed based on either surface deposition or pore filling. In the case of surface deposition, a template with a 3D interconnected network structure is synthesized first and the inorganic precursors are then deposited onto the surface of the template surface; finally, the template is removed, yielding an inorganic gel. Wang et al.[136] used swelling-induced mesoporous block copolymers (BCPs) as templates and atomic layer deposition (ALD) as the replication technique to synthesize networks of interconnected metal oxide nanotubes, including alumina oxide and titania oxide (Figure 14A). The prepared alumina nanotube networks consisted of continuous pore systems with high porosity ( 70-90\%) and ultrathin tube walls, with thicknesses down to $3 \mathrm{~nm}$. Ras et al.[137] used the ALD technique to deposit inorganic materials such as $\mathrm{TiO}_{2}$ and $\mathrm{ZnO}$ onto cellulose aerogels. After the removal of the cellulose via calcination, hollow inorganic nanotube aerogels were successfully synthesized. In the case of pore filling, interconnected channels are first prepared in a matrix, which is then filled with inorganic precursors to form an interconnected network. In the work of Rauber et 
al.,[138] a polymer foil was irradiated to form a 3D nanochannel network, which was then filled with the desired material, such as Au, Pt, or CdTe. After the removal of the polymer matrix, a freestanding 3D nanowire network was obtained. The size, shape, composition, and orientation of the nanowires and the integration level and three-dimensional complexity of the networks could all be controlled. Lu et al.[139] developed another route in which a film of mesoporous silica was coated onto a conductive substrate, the pore channels were then filled with metals or semiconductors via electrodeposition, and finally, the silica template was removed. A mesoporous nanowire network of Pt was successfully synthesized using this method.

The self-assembly of inorganic building blocks at the nano-level into macroscopic 3D architectures is an important bottom-up strategy in nano-research, bridging the gap between individual nanostructures and integrated structures. Among self-assembled 3D structured inorganic materials, functional gels have attracted widespread attention because the resulting gels typically exhibit hierarchical structures with controlled crystallinity and composition and therefore can inherit the chemical and physical features of nanomaterials in addition to the typical properties of macroporous materials. The dimensionality of nanoscale building blocks can vary from 0D to $1 \mathrm{D}$ and even 3D. For example, Lesnyak et al.[140] recently reported an efficient technique for assembling a variety of electrostatically stabilized all-inorganic semiconductor nanocrystals (NCs), including $\mathrm{CdSe}, \mathrm{PdSe}$, and $\mathrm{ZnO}$, by linking them into gel networks with appropriate ions. These all-inorganic non-ordered 3D assemblies exhibited tunable morphologies, compositions, sizes, and functional capping ligands. And their highly porous, monolithic structures preserved the quantum confinement of their building blocks. Benefiting from strong interparticle coupling, the gel facilitated charge transport between NCs, suggesting their potential for improved performance when used in energy conversion and storage applications. Kong et al.[141] synthesized hydrogels/aerogels via the in situ hydrothermal synthesis of $1 \mathrm{D}$ nanowires of $\mathrm{TiO}_{2}$ and $\mathrm{MnO}_{2}$ to directly form cross-linked networks during the synthesis process (Figure 14B). The density, porosity, and properties of these nanowire networks could be controlled by tuning the initial precursor concentration 
and the reaction time. The finely controlled aerogels showed remarkable porosities and surface areas, extremely low densities (as low as $2.9 \mathrm{mg} \mathrm{cm}^{-3}$ ), mechanical robustness, and superelasticity. Similarly, 2D nanomaterials can be also assembled into 3D networks. Sun et al.[142] prepared organic-free $\mathrm{MnO}_{2}$ aerogels with ultralow density via the self-assembly of $2 \mathrm{D} \mathrm{MnO}_{2}$ nanosheets using an ice-templating approach (Figure 14C). The large effective surfaces of 2D nanosheets for van der Waals attraction were critical to the successful formation of the aerogels, and the ice-squeezing effect helped to rearrange the squeezed nanosheets during assembly. The resulted aerogels exhibited densities as low as $0.53 \mathrm{mg} / \mathrm{cm}^{3}$, a strong oxidation ability and high porosity.

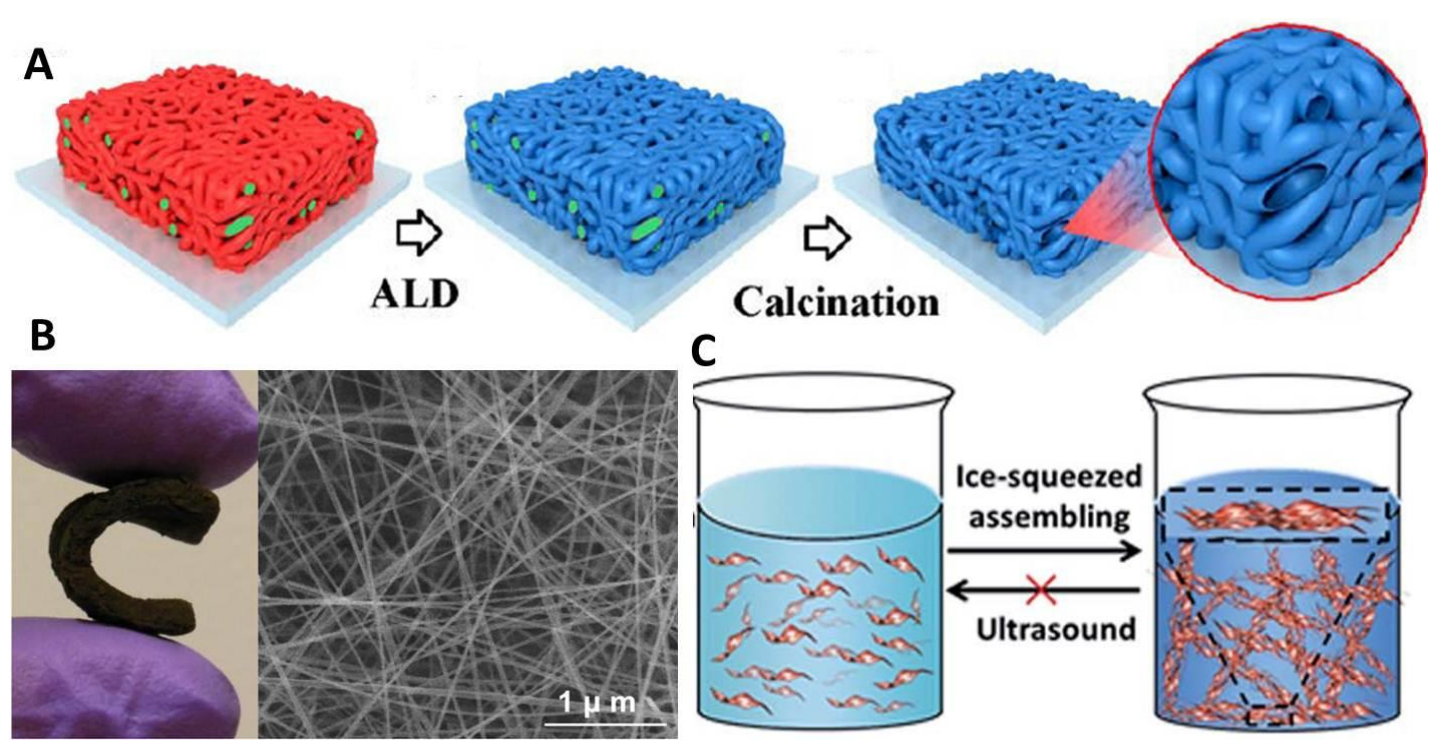

Figure 14. (A) Networks of interconnected metal oxide nanotubes synthesized by using swelling-induced mesoporous block copolymers (BCPs) as templates and atomic layer deposition (ALD) as the replication technique. Reproduced with permission from ref. 136. Copyright 2012 American Chemical Society. (B) Hydrogels/aerogels produced via the in situ hydrothermal synthesis of 1D nanowires of $\mathrm{TiO}_{2}$ and $\mathrm{MnO}_{2}$ to directly form cross-linked networks during the synthesis process. Reproduced with permission from ref. 141. Copyright 2014 American Chemical Society. (C) Organic-free $\mathrm{MnO}_{2}$ aerogels produced via the self-assembly of $2 \mathrm{D} \mathrm{MnO}_{2}$ nanosheets using an ice-templating approach. Reproduced with permission from ref. 142. Copyright 2016 Royal Society of Chemistry. 


\subsection{Perspective}

Although gel materials with 3D network structures have been synthesized using various inorganic materials and employed in applications such as catalysis, oil removal, and dye absorption, few studies on their application for energy conversion and storage have been reported. The reason may be that early research regarding inorganic gels primarily focused on metal and electrochemically non-active semiconductor materials. With the development of new synthesis methods and chemical modification strategies, more kinds of inorganic materials have been successfully assembled into gels, and it has become possible to delicately control their structures. These newly developed inorganic gels, such as $\mathrm{MnO}_{2}$ gels, can be directly used as electrode materials for supercapacitors. Previously synthesized inorganic gels can also find promising uses in energy-related applications. For example, metal gels can be used as transparent and flexible conductive electrodes in solar cells. Semiconductor gels, such as $\mathrm{ZnO}$ and $\mathrm{TiO}_{2}$ gels, can be used as electrodes in dye-sensitized solar cells and as electrochemical catalysts. We also believe that, as in the case of graphene, more gel-type materials will be invented based on electrochemically active inorganic materials and will play an important role in the development of high-performance energy devices.

\section{Conclusions \& Perspective}

Gel materials, as a special class of bio-inspired materials with structures or properties that mimic those of natural materials, are assembled from low-dimensional blocks (0D nanoparticles or clusters, 1D nanowires or polymer chains, 2D nanosheets or graphene sheets) and sometimes liquid phases to form complex hierarchical architectures. Regardless of the kind of blocks of which they are made, gel materials exhibit unique 3D network structures that endow them with unique chemical/physical properties, which fundamentally differentiate these materials from other low-dimensional nanomaterials. A gel maintains the mechanical integrity of the entire 
matrix, provides a large surface area to support a large number of reaction and anchoring sites, offers interconnected transport paths for electrons, and presents hierarchical pores to facilitate the diffusion of liquid and ions. These advantageous features make gel materials promising candidates for use in energy conversion and storage systems, in which the transport of electrons and ions, interfacial reactions, and reaction-induced stresses are generally involved [143].

To date, various synthesis strategies have been developed for the preparation of gel-based nanomaterials, including carbon-based gels, conductive polymer gels, ionically conductive gels and inorganic gels. These gel materials have successfully served as electrode materials, electrolytes, self-supported current collectors, 3D binder systems, etc. in various kinds of energy applications, such as LIBs, supercapacitors, catalysts, and fuel cells, showing excellent performance. Benefiting from the versatility of the $3 \mathrm{D}$ structures of gel materials, numerous approaches have been applied to improve their properties, including ionic/electronic conductivity, structure stability, interfacial reactivity/compatibility, and mechanical strength. The backbones of these gels can be chemically modified by grafting functional groups onto their surfaces, hybridizing them with functional molecules and nanoparticles, and/or introducing a second network to form a double-network structure. Chemical modification can introduce additional features, such as enhanced mechanical strength, even stretchability, stimuli-responsive properties and self-healing properties, thus providing a route toward novel functional energy devices, such as self-healing and/or responsive energy devices. In addition, the physical properties of gel materials, especially their mechanical properties, can also be controlled by tuning the parameters of their microstructures, including pore size, pore distribution, and the interspacing between building blocks. Inspired by their structure-derived mechanical properties, gel materials have been regarded as potential candidates for the fabrication of highly flexible and stretchable energy conversion and storage devices. The high scalability and processability of gel materials also endow them with the ability to be processed via ink-jet printing or spray coating, thereby enabling the large-scale and inexpensive fabrication of printable energy-storage devices. 
In future research, the chemical and physical properties of gel materials will need to be further tuned and modified via molecular level modification and nano-level processing. Different types of building blocks including electrochemically active inorganic nanomaterials, polymer semiconductors, and newly synthesized ionic polymers need to be developed to construct 3D structured gel materials. Other than present gel materials which usually use one kind of building materials, more complicated gel systems can be built up by combining different building blocks such as carbon materials and conductive polymers, inorganic nanomaterials and ionic polymers, as well as the combination of different kinds of polymers. These gel systems may combine the advantages of individual material and generate synergic effects, becoming multifunctional gel materials [144-149]. To achieve fine control of these hybrid gel systems, the chemical and physical interactions between different components should be well controlled and new crosslinking molecules need to be developed.

Based on the unique properties of gel materials, such as flexibility, stretchability, potential transparency, and biocompatibility, various types of novel energy devices can be developed. Highly flexible and stretchable solar cells, batteries, and supercapacitors can be created by coating gel materials onto flexible substrates or directly combining gel components into free-standing electrodes. Smart energy devices can be built by using responsive polymer gels which could respond to environmental stimuli. More importantly, with help of energy gels, the applications of energy devices could be extended to other technological areas. For example, artificial skins and flexible biosensors can be developed by using gel electrodes and electrolyte. Micro energy devices can be also developed with the flexibility and decent processing ability of gel materials.

These abovementioned achievements will rely on fundamental studies and advanced characterization techniques, which will be essential to help researchers gain a deeper understanding of various aspects of gel materials, including the relationships between nano-/microstructural and mechanical properties, the effects of building-block assembly and surface chemistry on charge transport, and the 
electrochemical dynamics at the interfaces between gel backbones and electrolytes. Through such interdisciplinary research, more and more promising gel materials with advantageous performance for energy conversion and storage applications will be developed.

\section{Acknowledgement}

G.Y. acknowledges the Center for Mesoscale Transport Properties, an Energy Frontier Research Center supported by the U.S. Department of Energy, Office of Science, Basic Energy Sciences, under award \#DE-SC0012673 for financial support. G.Y. also acknowledges the financial support from National Science Foundation award NSF-CMMI-1537894. L.P. and Y.S. are thankful for financial support from Chinese National Key Fundamental Research Project (2013CB932900), National Natural Science Foundation of China (61229401, 61076017, 60990314), and PAPD program. 


\section{References}

[1] K.Q. Peng, X. Wang, L. Li, Y. Hu, S.T. Lee, Nano Today 8 (2013) 75-97.

[2] M.R. Gao, Y.F. Xu, J. Jiang, S.H. Yu, Chem. Soc. Rev. 42 (2013) 2986-3017.

[3] L. Dai, Acc. Chem. Res. 46 (2013) 31-42.

[4] Y. Shi, G. Yu, Chem. Mater. 28 (2016) 2466-2477.

[5] J.B. Goodenough, K.S. Park, J. Am. Chem. Soc. 135 (2013) 1167-1176.

[6] G. Wang, L. Zhang, J. Zhang, Chem. Soc. Rev. 41 (2012) 797-828.

[7] Q. Zhang, E. Uchaker, S.L. Candelaria, G. Cao, Chem. Soc. Rev. 42 (2013) 3127-3171.

[8] S. Nardecchia, D. Carriazo, M.L. Ferrer, M.C. Gutierrez, F. del Monte, Chem. Soc. Rev. 42 (2013) 794-830.

[9] J. Xu, X. Wang, X. Wang, D. Chen, X. Chen, D. Li, G. Shen, ChemElectroChem 1 (2014) 975-1002.

[10] Y. Xiang, S. Lu, S.P. Jiang, Chem. Soc. Rev. 41 (2012) 7291-7321.

[11] Y. Shi, L. Peng, Y. Ding, Y. Zhao, G. Yu, Chem. Soc. Rev. 44 (2015) 6684-6696.

[12] K. Deligkaris, T.S. Tadele, W. Olthuis, A. van den Berg, Sensor. Acuat. B Chem. 147 (2010) 765-774.

[13] C.H. Zhu, Y. Lu, J.F. Chen, S.H. Yu, Small (2014) 2796-2800.

[14] H.W. Liang, Q.F. Guan, L.F. Chen, Z. Zhu, W.J. Zhang, S.H. Yu, Angew. Chem. Int. Ed. 51 (2012) 5101-5105.

[15] J. Ge, H.B. Yao, X. Wang, Y.D. Ye, J.L. Wang, Z.Y. Wu, J.W. Liu, F.J. Fan, H.L. Gao, C.L. Zhang, S.H. Yu, Angew. Chem. Int. Ed. 125 (2013) 1698-1703.

[16] H.P. Cong, P. Wang, S.H. Yu, Chem. Mater. 25 (2013) 3357-3362.

[17] R. Chakrabarty, P.S. Mukherjee, P.J. Stang, Chem. Rev. 111 (2011) 6810-6918. 
[18] N.A. Peppas, J.Z. Hilt, A. Khademhosseini, R. Langer, Adv. Mater. 18 (2006) 1345-1360.

[19] J. Lu, F. Yan, J. Texter, Prog. Polym. Sci. 34 (2009) 431-448.

[20] M.B. Bryning, D.E. Milkie, M.F. Islam, L.A. Hough, J.M. Kikkawa, A.G. Yodh, Adv. Mater. 19 (2007) 661-664.

[21] J. Zou, J. Liu, A.S. Karakoti, A. Kumar, D. Joung, Q. Li, S.I. Khondaker, S. Seal, L. Zhai, ACS Nano 4 (2010) 7293-7302.

[22] K. Chen, S. Song, F. Liu, D. Xue, Chem. Soc. Rev. 44 (2015) 6230-6257.

[23] V. Chabot, D. Higgins, A. Yu, X. Xiao, Z. Chen, J. Zhang, Energy Environ. Sci. (2014) 1564-1596.

[24] Y. Xu, K. Sheng, C. Li, G. Shi, ACS Nano 4 (2010) 4324-4330.

[25] H. Bai, C. Li, X. Wang, G. Shi, J. Phys. Chem. C 115 (2011) 5545-5551.

[26] F. Liu, T.S. Seo, Adv. Funct. Mater. 20 (2010) 1930-1936.

[27] B. Adhikari, A. Banerjee, Soft Matter 7 (2011) 9259-9266.

[28] Q.Y. Cheng, D. Zhou, Y. Gao, Q. Chen, Z. Zhang, B.H. Han, Langmuir 28 (2012) 3005-3010.

[29] Y. Xu, Q. Wu, Y. Sun, H. Bai, G. Shi, ACS Nano 4 (2010) 7358-7362.

[30] C. Huang, H. Bai, C. Li, G. Shi, Chem. Commun. 47 (2011) 4962-4964.

[31] H.P. Cong, P. Wang, S.H. Yu, Small 10 (2014) 448-453.

[32] H.P. Cong, X.C. Ren, P. Wang, S.H. Yu, ACS Nano 6 (2012) 2693-2703.

[33] F. Yavari, Z. Chen, A.V. Thomas, W. Ren, H.M. Cheng, N. Koratkar, Sci. Rep. 1 (2011) 166.

[34] X. Xie, G. Yu, N. Liu, Z. Bao, C.S. Criddle, Y. Cui, Energy Environ. Sci. 5 (2012) 6862-6866. 
[35] D.D. Nguyen, N.H. Tai, S.B. Lee, W.S. Kuo, Energy Environ. Sci. 5 (2012) 7908-7912.

[36] L. Zhang, G. Shi, J. Phys. Chem. C 115 (2011) 17206-17212.

[37] Y. Xu, Z. Lin, X. Huang, Y. Liu, Y. Huang, X. Duan, ACS Nano 7 (2013) 4042-4049.

[38] Y. Xu, Z. Lin, X. Huang, Y. Wang, Y. Huang, X. Duan, Adv. Mater. (2013) $5779-5784$.

[39] Y. Xu, Z. Lin, X. Zhong, X. Huang, N.O. Weiss, Y. Huang, X. Duan, Nat. Commun. 5 (2014) 4554.

[40] P. Chen, J.J. Yang, S.S. Li, Z. Wang, T.Y. Xiao, Y.H. Qian, S.H. Yu, Nano Energy 2 (2013) 249-256.

[41] Z.S. Wu, A. Winter, L. Chen, Y. Sun, A. Turchanin, X. Feng, K. Müllen, Adv. Mater. 24 (2012) 5130-5135.

[42] U.N. Maiti, J. Lim, K.E. Lee, W.J. Lee, S.O. Kim, Adv. Mater. 26 (2014) 615-619.

[43] H. Gao, F. Xiao, C.B. Ching, H. Duan, ACS Appl. Mater. Interfaces 4 (2012) 2801-2810.

[44] S. Chen, W. Yeoh, Q. Liu, G. Wang, Carbon 50 (2012) 4557-4565.

[45] Z. Jiang, B. Pei, A. Manthiram, J. Mater. Chem. A 1 (2013) 7775-7781.

[46] B. Wang, W. Al Abdulla, D. Wang, X.S. Zhao, Energy Environ. Sci. 8 (2015) 869-875.

[47] Y. Gong, S. Yang, Z. Liu, L. Ma, R. Vajtai, P.M. Ajayan, Adv. Mater. 25 (2013) 3979-3984.

[48] F. Zhang, X. Yang, Y. Xie, N. Yi, Y. Huang, Y. Chen, Carbon 82 (2015) 161-167.

[49] S. Lu, Y. Chen, X. Wu, Z. Wang, Y. Li, Sci. Rep. 4 (2014) 4629. 
[50] C.M. Xu, Y.S. Wu, X.Y. Zhao, X.L. Wang, G.H. Du, J. Zhang, J.P. Tu, J. Power Sources 275 (2015) 22-25.

[51] S. Chen, J. Duan, M. Jaroniec, S.-Z. Qiao, Adv. Mater. 26 (2014) 2925-2930.

[52] S. Chen, J. Duan, Y. Tang, B. Jin, S. Zhang Qiao, Nano Energy 11 (2015) 11-18.

[53] Z.-S. Wu, S. Yang, Y. Sun, K. Parvez, X. Feng, K. Müllen, J. Am. Chem. Soc. 134 (2012) 9082-9085.

[54] G. Giri, E. Verploegen, S.C.B. Mannsfeld, S. Atahan-Evrenk, D.H. Kim, S.Y. Lee, H.A. Becerril, A. Aspuru-Guzik, M.F. Toney, Z. Bao, Nature 480 (2011) 504-508.

[55] J. Kopecek, Nature 417 (2002) 388-391.

[56] A.I. Horowitz, M.J. Panzer, J. Mater. Chem. 22 (2012) 16534-16539.

[57] H. Bai, K. Sheng, P. Zhang, C. Li, G. Shi, J. Mater. Chem. 21 (2011) 18653-18658.

[58] Y. Zhao, B. Liu, L. Pan, G. Yu, Energy Environ. Sci. 6 (2013) 2856-2870.

[59] L. Pan, A. Chortos, G. Yu, Y. Wang, S. Isaacson, R. Allen, Y. Shi, R. Dauskardt, Z. Bao, Nat. Commun. 5 (2014) 3002.

[60] Y. Shi, L. Pan, B. Liu, Y. Wang, Y. Cui, Z. Bao, G. Yu, J. Mater. Chem. A 2 (2014) 6086-6091.

[61] Y. Wang, Y. Shi, L. Pan, Y. Ding, Y. Zhao, Y. Li, Y. Shi, G. Yu, Nano Lett. 15 (2015) 7736-7741.

[62] P. Novák, K. Müller, K.S.V. Santhanam, O. Haas, Chem. Rev. 97 (1997) 207-282.

[63] K. Kirshenbaum, D.C. Bock, C.Y. Lee, Z. Zhong, K.J. Takeuchi, A.C. Marschilok, E.S. Takeuchi, Science 347 (2015) 149-154.

[64] M. Wu, X. Xiao, N. Vukmirovic, S. Xun, P.K. Das, X. Song, P. Olalde-Velasco, D. Wang, A.Z. Weber, L.W. Wang, V.S. Battaglia, W. Yang, G. Liu, J. Am. Chem. Soc. 
135 (2013) 12048-12056.

[65] H. Wu, G. Yu, L. Pan, N. Liu, M.T. McDowell, Z. Bao, Y. Cui, Nat. Commun. 4 (2013) 1943.

[66] B. Liu, P. Soares, C. Checkles, Y. Zhao, G. Yu, Nano Lett. 13 (2013) 3414-3419.

[67] Y. Tang, Y. Zhang, W. Li, B. Ma, X. Chen, Chem. Soc. Rev. 44 (2015) 5926-5940.

[68] J.W.F. To, Z. Chen, H. Yao, J. He, K. Kim, H.-H. Chou, L. Pan, J. Wilcox, Y. Cui, Z. Bao, ACS Centr. Sci. 1 (2015) 68-76.

[69] J. Zhang, Z. Zhao, Z. Xia, L. Dai, Nat. Nanotechnol. 10 (2015) 444-452.

[70] W. Ding, L. Li, K. Xiong, Y. Wang, W. Li, Y. Nie, S. Chen, X. Qi, Z. Wei, J. Am. Chem. Soc. 137 (2015) 5414-5420.

[71] N. Yoshimoto, Gel Electrolyte for Supercapacitors, Nonaqueous, in: G. Kreysa, K.-i. Ota, R.F. Savinell (Eds.) Encyclopedia of Applied Electrochemistry, Springer New York, New York, NY, 2014, pp. 936-939.

[72] M. Marcinek, J. Syzdek, M. Marczewski, M. Piszcz, L. Niedzicki, M. Kalita, A. Plewa-Marczewska, A. Bitner, P. Wieczorek, T. Trzeciak, M. Kasprzyk, P. Lezak, Z. Zukowska, A. Zalewska, W. Wieczorek, Solid State Ionics 276 (2015) 107-126.

[73] N.A. Choudhury, S. Sampath, A.K. Shukla, Energy Environ. Sci. 2 (2009) 55-67.

[74] J.P. Gong, Y. Katsuyama, T. Kurokawa, Y. Osada, Adv. Mater. 15 (2003) $1155-1158$.

[75] F. Luo, T.L. Sun, T. Nakajima, T. Kurokawa, Y. Zhao, K. Sato, A.B. Ihsan, X. Li, H. Guo, J.P. Gong, Adv. Mater. 27 (2015) 2722-2727.

[76] J.P. Gong, Science 344 (2014) 161-162.

[77] J.Y. Sun, X. Zhao, W.R.K. Illeperuma, O. Chaudhuri, K.H. Oh, D.J. Mooney, J.J. Vlassak, Z. Suo, Nature 489 (2012) 133-136. 
[78] C. Keplinger, J.Y. Sun, C.C. Foo, P. Rothemund, G.M. Whitesides, Z.G. Suo, Science 341 (2013) 984-987.

[79] Y.Y. Bai, B.H. Chen, F. Xiang, J.X. Zhou, H. Wang, Z.G. Suo, Appl. Phys. Lett. 105 (2014) 151903.

[80] T.L. Sun, T. Kurokawa, S. Kuroda, A.B. Ihsan, T. Akasaki, K. Sato, M.A. Haque, T. Nakajima, J.P. Gong, Nat. Mater. 12 (2013) 932-937.

[81] G. Feuillade, P. Perche, J. Appl. Electrochem. 5 (1975) 63-69.

[82] J.M. Tarascon, A.S. Gozdz, C. Schmutz, F. Shokoohi, P.C. Warren, Solid State Ionics 86-88, Part 1 (1996) 49-54.

[83] W. Pu, X. He, L. Wang, C. Jiang, C. Wan, J. Membrane Sci. 272 (2006) 11-14.

[84] S.W. Choi, S.M. Jo, W.S. Lee, Y.R. Kim, Adv. Mater. 15 (2003) 2027-2032.

[85] Y. Zhu, F. Wang, L. Liu, S. Xiao, Z. Chang, Y. Wu, Energy Environ. Sci. 6 (2013) 618-624.

[86] S. Zhang, K.H. Lee, J. Sun, C.D. Frisbie, T.P. Lodge, Macromolecules 44 (2011) 8981-8989.

[87] J. Le Bideau, L. Viau, A. Vioux, Chem. Soc. Rev. 40 (2011) 907-925.

[88] M.J. Park, I. Choi, J. Hong, O. Kim, J. Appl. Polym. Sci. 129 (2013) 2363-2376.

[89] M. Kofu, T. Someya, S. Tatsumi, K. Ueno, T. Ueki, M. Watanabe, T. Matsunaga, M. Shibayama, V.G. Sakai, M. Tyagi, O. Yamamuro, Soft Matter 8 (2012) 7888-7897.

[90] Y. Wang, Y. Chen, J.W. Gao, H.G. Yoon, L.Y. Jin, M. Forsyth, T.J. Dingemans, L.A. Madsen, Adv. Mater. 28 (2016) 2571-2578.

[91] Y. Kitazawa, K. Iwata, S. Imaizumi, H. Ahn, S.Y. Kim, K. Ueno, M.J. Park, M. Watanabe, Macromolecules 47 (2014) 6009-6016.

[92] O. Kim, G. Jo, Y.J. Park, S. Kim, M.J. Park, J. Phys. Chem. Lett. 4 (2013) 2111-2117. 
[93] C. Zhong, Y. Deng, W. Hu, J. Qiao, L. Zhang, J. Zhang, Chem. Soc. Rev. 44 (2015) 7484-7539.

[94] X. Lu, M. Yu, G. Wang, Y. Tong, Y. Li, Energy Environ. Sci. 7 (2014) 2160-2181.

[95] J. Kalupson, D. Ma, C.A. Randall, R. Rajagopalan, K. Adu, J. Phys. Chem. C 118 (2014) 2943-2952.

[96] M.J. Jiang, J.D. Zhu, C. Chen, Y. Lu, Y.Q. Ge, X.W. Zhang, ACS Appl. Mater. Interfaces 8 (2016) 3473-3481.

[97] H. Fei, C. Yang, H. Bao, G. Wang, J. Power Sources 266 (2014) 488-495.

[98] H. Yu, J. Wu, L. Fan, Y. Lin, K. Xu, Z. Tang, C. Cheng, S. Tang, J. Lin, M. Huang, Z. Lan, J. Power Sources 198 (2012) 402-407.

[99] C. Yang, M. Sun, X. Wang, G. Wang, ACS Sustain. Chem. Eng. 3 (2015) 2067-2076.

[100] M. Suleman, Y. Kumar, S.A. Hashmi, Mater. Chem. Phys. 163 (2015) 161-171.

[101] M. Le Thai, G.T. Chandran, R.K. Dutta, X. Li, R.M. Penner, ACS Energy Lett. (2016) 57-63.

[102] Y.J. Kang, S.J. Chun, S.S. Lee, B.Y. Kim, J.H. Kim, H. Chung, S.Y. Lee, W. Kim, ACS Nano 6 (2012) 6400-6406.

[103] G. Lee, D. Kim, D. Kim, S. Oh, J. Yun, J. Kim, S.S. Lee, J.S. Ha, Energy Environ. Sci. 8 (2015) 1764-1774.

[104] P. Thoniyot, M.J. Tan, A.A. Karim, D.J. Young, X.J. Loh, Adv. Sci. 2 (2015) 1400010 .

[105] Y. Huang, M. Zhong, Y. Huang, M.S. Zhu, Z.X. Pei, Z.F. Wang, Q. Xue, X.M. Xie, C.Y. Zhi, Nat. Commun. 6 (2015) 10310.

[106] X. Liu, D. Wu, H. Wang, Q. Wang, Adv. Mater. 26 (2014) 4370-4375. 
[107] A. Manuel Stephan, Eur. Polym. J. 42 (2006) 21-42.

[108] E. Quartarone, P. Mustarelli, Chem. Soc. Rev. 40 (2011) 2525-2540.

[109] Q.Q. Tang, M.M. Chen, C.Y. Yang, W.Q. Wang, H. Bao, G.C. Wang, ACS Appl. Mater. Interfaces 7 (2015) 15303-15313.

[110] K.M. Kim, M. Latifatu, Y.G. Lee, J.M. Ko, J.H. Kim, W.I. Cho, J. Electroceram. 32 (2014) 146-153.

[111] W.K. Shin, J. Cho, A.G. Kannan, Y.S. Lee, D.W. Kim, Sci. Rep. 6 (2016) 26332.

[112] W.K. Shin, J.H. Yoo, W. Choi, K.Y. Chung, S.S. Jang, D.W. Kim, J. Mater. Chem. A 3 (2015) 12163-12170.

[113] C.L. Cheng, C.C. Wan, Y.Y. Wang, J. Power Sources 134 (2004) 202-210.

[114] Q. Lu, J. Yang, W. Lu, J. Wang, Y. Nuli, Electrochim. Acta 152 (2015) 489-495.

[115] H.Y. Wu, D. Saikia, H.Y. Chao, Y.C. Pan, J. Fang, L.D. Tsai, G.T.K. Fey, H.M. Kao, J. Power Sources 238 (2013) 265-273.

[116] W.W. Huang, Z.Q. Zhu, L.J. Wang, S.W. Wang, H. Li, Z.L. Tao, J.F. Shi, L.H. Guan, J. Chen, Angew. Chem. Int. Ed. 52 (2013) 9162-9166.

[117] C.V. Amanchukwu, J.R. Harding, Y. Shao-Horn, P.T. Hammond, Chem. Mater. 27 (2015) 550-561.

[118] D.J. Lee, H. Lee, Y.J. Kim, J.K. Park, H.T. Kim, Adv. Mater. 28 (2016) 857-863.

[119] W. Xu, J. Wang, F. Ding, X. Chen, E. Nasybulin, Y. Zhang, J.G. Zhang, Energy Environ. Sci. 7 (2014) 513-537.

[120] W.K. Shin, S.M. Park, Y.S. Lee, D.W. Kim, J. Electrochem. Soc. 162 (2015) A2628-A2634.

[121] T. Zhang, H.S. Zhou, Angew. Chem. Int. Ed. 51 (2012) 11062-11067.

[122] W. Zhai, H.J. Zhu, L. Wang, X.M. Liu, H. Yang, Electrochim. Acta 133 (2014) 623-630. 
[123] L. Yang, J.Y. Hu, G. Lei, H.T. Liu, Chem. Eng. J. 258 (2014) 320-326.

[124] A. Yella, H.W. Lee, H.N. Tsao, C. Yi, A.K. Chandiran, M.K. Nazeeruddin, E.W.G. Diau, C.Y. Yeh, S.M. Zakeeruddin, M. Grätzel, Science 334 (2011) 629-634.

[125] P. Wang, S.M. Zakeeruddin, J.E. Moser, M.K. Nazeeruddin, T. Sekiguchi, M. Gratzel, Nat. Mater. 2 (2003) 402-407.

[126] C.L. Chen, H. Teng, Y.L. Lee, Adv. Mater. 23 (2011) 4199-4204.

[127] F. Bella, N. Vlachopoulos, K. Nonomura, S.M. Zakeeruddin, M. Gratzel, C. Gerbaldi, A. Hagfeldt, Chem. Commun. 51 (2015) 16308-16311.

[128] R.X. Dong, S.Y. Shen, H.W. Chen, C.C. Wang, P.T. Shih, C.T. Liu, R. Vittal, J.J. Lin, K.C. Ho, J. Mater. Chem. A 1 (2013) 8471-8478.

[129] M.H. Khanmirzaei, S. Ramesh, K. Ramesh, Sci. Rep. 5 (2015) 18056.

[130] K.S. Lee, Y. Jun, J.H. Park, Nano Lett. 12 (2012) 2233-2237.

[131] N.A. Choudhury, J. Ma, Y. Sahai, J. Power Sources 210 (2012) 358-365.

[132] N. Plumeré, O. Rüdiger, A.A. Oughli, R. Williams, J. Vivekananthan, S. Pöller, W. Schuhmann, W. Lubitz, Nat. Chem. 6 (2014) 822-827.

[133] B.P. Tripathi, M. Kumar, V.K. Shahi, J. Membrane. Sci. 360 (2010) 90-101.

[134] S.Y. Lee, T. Yasuda, M. Watanabe, J. Power Sources 195 (2010) 5909-5914.

[135] A.E. Gash, T.M. Tillotson, J.H. Satcher Jr, L.W. Hrubesh, R.L. Simpson, J. Non-Cryst. Solids 285 (2001) 22-28.

[136] F. Li, X. Yao, Z. Wang, W. Xing, W. Jin, J. Huang, Y. Wang, Nano Lett. 12 (2012) 5033-5038.

[137] J.T. Korhonen, P. Hiekkataipale, J. Malm, M. Karppinen, O. Ikkala, R.H.A. Ras, ACS Nano 5 (2011) 1967-1974.

[138] M. Rauber, I. Alber, S. Müller, R. Neumann, O. Picht, C. Roth, A. Schökel, M.E. Toimil-Molares, W. Ensinger, Nano Lett. 11 (2011) 2304-2310. 
[139] D. Wang, H. Luo, R. Kou, M.P. Gil, S. Xiao, V.O. Golub, Z. Yang, C.J. Brinker, Y. Lu, Angew. Chem. Int. Ed. 43 (2004) 6169-6173.

[140] V. Sayevich, B. Cai, A. Benad, D. Haubold, L. Sonntag, N. Gaponik, V. Lesnyak, A. Eychmüller, Angew. Chem. Int. Ed. 55 (2016) 6334-6338.

[141] S.M. Jung, H.Y. Jung, W. Fang, M.S. Dresselhaus, J. Kong, Nano Lett. 14 (2014) $1810-1817$.

[142] Z. Liu, K. Xu, P. She, S. Yin, X. Zhu, H. Sun, Chem. Sci. 7 (2016) 1926-1932.

[143] L. Luo, P. Zhao, H. Yang, B. Liu, J.G. Zhang, Y. Cui, G. Yu, S. Zhang, C.M. Wang, Nano Lett. 15 (2015) 7016-7022.

[144] Y. Shi, M. Wang, C. Ma, Y. Wang, X. Li, G. Yu, Nano Lett. 15 (2015) 6276-6281.

[145] C. Ma, Y. Shi, D.A. Pena, L. Peng, G. Yu, Angew. Chem. 127 (2015) 7484-7488.

[146] L. Li, Y. Wang, L. Pan, Y. Shi, W. Cheng, Y. Shi, G. Yu, Nano Lett. 15 (2015) $1146-1151$.

[147] Y. Shi, H. Ha, A. Al-Sudani, C.J. Ellison, G. Yu, Adv. Mater. 28 (2016) DOI: 10.1002/adma.201602239.

[148] Y. Shi, C. Ma, L. Peng, G. Yu, Adv. Funct. Mater. 25 (2015) 1219-1225.

[149] Y. Wang, Y. Shi, L. Pan, M. Yang, L. Peng, S. Zong, Y. Shi, G. Yu, Nano Lett. 14 (2014) 4803-4809. 

Table of contents

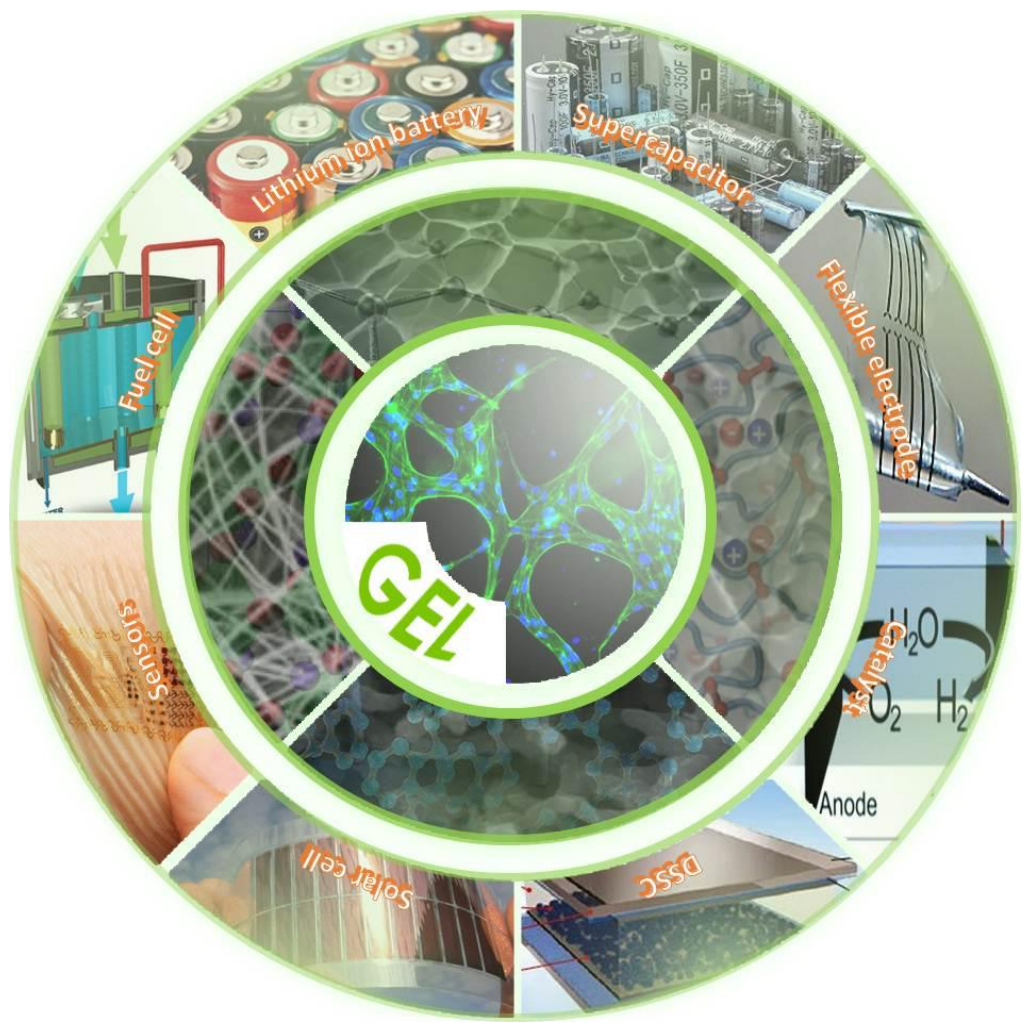

